JOURNAL OF

FUNCTION SPACES AND APPLICATIONS

Volume 3, Number 3 (2005), 287-320 (c) 2005, Scientific Horizon

http://www.jfsa.net

\title{
Box dimension, oscillation and smoothness in function spaces*
}

\author{
Abel Carvalho \\ (Communicated by Hans Triebel)
}

2000 Mathematics Subject Classification. 46E35, $28 \mathrm{~A} 80$.

Keywords and phrases. Smoothness, Besov spaces, Oscillation spaces, Box dimension.

\footnotetext{
Abstract. The aim of this paper is twofold. First we relate upper and lower box dimensions with oscillation spaces, and we develop embeddings or inclusions between oscillation spaces and Besov spaces. Secondly, given a point in the $\left(\frac{1}{p}, s\right)$ plane we determine maximal and minimal values for the upper box dimension (also the maximal value for lower box dimension) for the graphs of continuous real functions with a compact support, represented by this point.
}

\footnotetext{
${ }^{*}$ Research partially supported by Gabinete de Relações Internacionais da Ciência e do Ensino Superior and Deutscher Akademischer Austauschdienst (Convénio GRICESDAAD) and by the Junior Research Team 'Fractal Analysis' of the University in Jena.

Also partially supported by Unidade de Investigação Matemática e Aplicações of Universidade de Aveiro through Programa Operacional 'Ciência, Tecnologia, Inovação' (POCTI) of the Fundação para a Ciência e a Tecnologia (FCT), cofinanced by the European Community fund (FEDER).
} 


\section{Introduction}

The filling of the gaps between spaces, giving origin to the concept of smoothness for any real $s$, was an important step in the systematization of the function spaces. In particular, since the Sixties of the last century there are many papers about Besov spaces $B_{p, q}^{s}\left(\mathbb{R}^{n}\right)$ and Triebel-Lizorkin spaces $F_{p, q}^{s}\left(\mathbb{R}^{n}\right)$. The diversification of techniques to measure smoothness, motivated by theoretical and practical reasons, has been essential in this view of unification.

Let $n \in \mathbb{N}$. In what follows we are looking for functions $f: \mathbb{R}^{n} \rightarrow \mathbb{R}$ continuous and with a compact support, denoting this condition by (CCS). We define graph of $f$ by

$$
\Gamma(f)=\left\{(x, f(x)): x \in K_{f}=\left[-k_{f}, k_{f}\right]^{n}\right\},
$$

where $k_{f} \in \mathbb{N}$ is chosen such that supp $f \subset K_{f}$. We want to relate three different forms of measuring smoothness of continuous real functions with a compact support:

$\triangleright$ Frequency structure, concerning the Besov spaces $B_{p, p}^{s}\left(\mathbb{R}^{n}\right)$ that contain $f$ or not,

$\triangleright$ Box dimension, that deals with the minimum number of cubes needed to cover $\Gamma(f)$,

$\triangleright$ Oscillations, the sum of differences between local maximums and minimums of $f$.

Let $0<p \leq \infty, 0<q \leq \infty$ and $s \in \mathbb{R}$. The frequency structure of the functions $f$ is characterized by the Besov spaces $B_{p, q}^{s}\left(\mathbb{R}^{n}\right)$ and Triebel-Lizorkin spaces $F_{p, q}^{s}\left(\mathbb{R}^{n}\right)(0<p<\infty)$. We would like to recall (see [9], 2.3.5) that these contain as special cases the Bessel potential spaces $H_{p}^{s}\left(\mathbb{R}^{n}\right)=F_{p, 2}^{s}\left(\mathbb{R}^{n}\right)$ if $1<p<\infty$, Hölder-Zygmund spaces $\mathscr{C}^{s}\left(\mathbb{R}^{n}\right)=B_{\infty, \infty}^{s}\left(\mathbb{R}^{n}\right)$ if $s>0$, and Hölder spaces $C^{s}\left(\mathbb{R}^{n}\right)=B_{\infty, \infty}^{s}\left(\mathbb{R}^{n}\right)$ if $0<s \neq$ integer.

Consider now $0<p \leq \infty$ and $s>0$, and suppose that $f$ verify the condition (CCS). Then by definition, $f$ belongs to the set $B_{p}^{s *}\left(\mathbb{R}^{n}\right)$ if and only if for all $\varepsilon>0$ we have

$$
f \in B_{p, p}^{s-\varepsilon}\left(\mathbb{R}^{n}\right)
$$

and

$$
f \notin B_{p, p}^{s+\varepsilon}\left(\mathbb{R}^{n}\right) .
$$

We use a graphical representation in the $\left(\frac{1}{p}, s\right)$-plane, interpreted in the $s *$ sense, i.e., point $\left(\frac{1}{p}, s\right)$ symbolizes all functions that belong to $B_{p}^{s *}\left(\mathbb{R}^{n}\right)$. Given $f \notin C^{\infty}\left(\mathbb{R}^{n}\right)$ satisfying (CCS), we define $s_{f}(t)$ such that 
$f \in B_{p}^{s_{f}(t) *}\left(\mathbb{R}^{n}\right)$, where $t=\frac{1}{p} \geq 0$. Then, the graph of $s_{f}(t)$ describes (see [12], p. 414) a continuous, concave and non-decreasing curve with left hand derivative $s_{f-}^{\prime}(t) \leq n$ for $t>0$, as represented in Figure 1 .

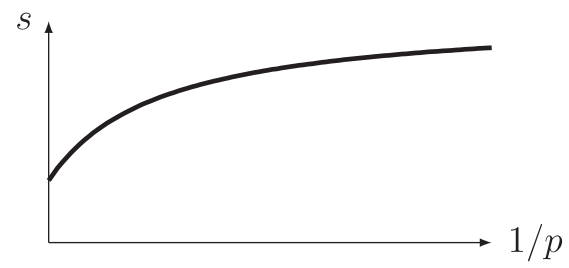

Figure 1. Tipical curve $s_{f}(t)$ for $t \geq 0$, where $f \notin C^{\infty}\left(\mathbb{R}^{n}\right)$ is a function satisfying (CCS)

The definition of $B_{p}^{s *}\left(\mathbb{R}^{n}\right)$ will be empty if $s<0$, since follows by elementary calculations that if $f$ satisfies the condition (CCS) then $f \in$ $B_{p, p}^{-\varepsilon}\left(\mathbb{R}^{n}\right), \forall \varepsilon>0$. But we restrict ourselves to $s>0$, thus having by the following Theorem 1.1 a non-empty definition.

Theorem 1.1 ([13], p. 202, Corollary 9 with elementary adaptations). Let $s>0$ and $f_{s}$ as in [13], p. 202. Then $f_{s} \in B_{p}^{s *}\left(\mathbb{R}^{n}\right)$ for $0<p \leq \infty$.

With corresponding notation applied to other spaces for $s>0$, we have the equalities $F_{p}^{s *}\left(\mathbb{R}^{n}\right)=B_{p}^{s *}\left(\mathbb{R}^{n}\right)$ if $0<p<\infty, H_{p}^{s *}\left(\mathbb{R}^{n}\right)=B_{p}^{s *}\left(\mathbb{R}^{n}\right)$ if $1<p<\infty$, and $C^{s *}\left(\mathbb{R}^{n}\right)=\mathscr{C}^{s *}\left(\mathbb{R}^{n}\right)=B_{\infty}^{s *}\left(\mathbb{R}^{n}\right)$, giving us a good reason to restrict the attention to Besov spaces.

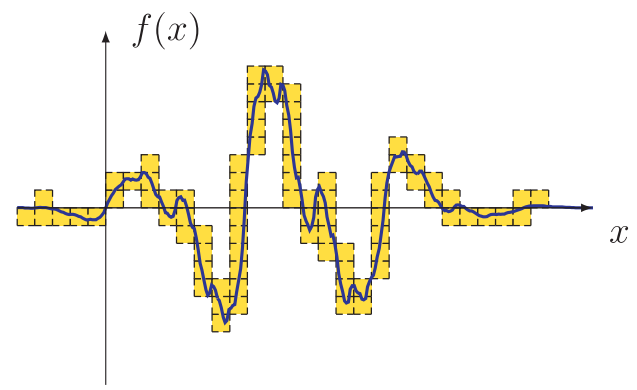

Figure 2. Graph of $f$ with a covering by small dyadic squares

Let us introduce some concepts about fractal geometry, which we want to relate with the function spaces. Suppose $f$ satisfying the condition (CCS) and let $N(\nu, f)$ be the minimum number of dyadic cubes of length side $2^{-\nu}$ in $\mathbb{R}^{n+1}$ required to cover $\Gamma(f)$ (see Figure 2 for the case $n=1$ ). Then we define the upper box dimension by

$$
\overline{\operatorname{dim}}_{B} \Gamma(f)=\varlimsup_{\nu \rightarrow \infty} \frac{\log _{2} N(\nu, f)}{\nu} .
$$


The lower box dimension is defined in a similar way but with the lower limit. The oscillations of the graph of a function play an important role in determination of the box dimension. If $I$ is a dyadic cube of $\mathbb{R}^{n}$ we define the oscillation of $f$ over $I$ by $\operatorname{osc}_{I}(f)=\sup _{I} f-\inf _{I} f$. Given a real number $\alpha$, the oscillation space $V^{\alpha}\left(\mathbb{R}^{n}\right)$ contains all real continuous functions with $\sup _{\nu \geq 0} 2^{\nu(\alpha-n)} \sum_{|I|=2^{-\nu n}} \operatorname{osc}_{I}(f)<\infty$.

The main aim of this paper is to calculate the maximal and minimal dimensions $\overline{\operatorname{Dim}}_{p}^{s}$ and $\overline{\operatorname{dim}}_{p}^{s}$ for $0<p \leq \infty$ and $s>0$. We define the maximal dimension by

$$
\overline{\operatorname{Dim}}_{p}^{s}=\sup _{f \in B_{p}^{s *}\left(\mathbb{R}^{n}\right)} \overline{\operatorname{dim}}_{B} \Gamma(f),
$$

and the minimal dimension is similarly defined by $\overline{\operatorname{dim}}_{p}^{s}=\inf _{f \in B_{p}^{s *(}\left(\mathbb{R}^{n}\right)} \overline{\operatorname{dim}}_{B} \Gamma(f)$.

Theorem 1.2. Let $s>0$ and let $f: \mathbb{R} \rightarrow \mathbb{R}$ be continuous and with a compact support. Suppose that $f \in B_{1}^{s *}(\mathbb{R})$. Then we have $\overline{\operatorname{dim}}_{B} \Gamma(f) \geq 2-\min (1, s)$.

The above Theorem provides a lower bound for the minimal dimension if $n=1, p=1$ and $s>0$. In [2], p. 220 we find an analogous result but, as we will see, both Theorem 1.2 and the counterpart in [2], p. 220 cannot be improved by an estimate from above.

The structure of the paper is the following. In the Section 2 we introduce the Besov spaces and the class $s *$. In Section 3 we define upper and lower box dimensions and in Section 4 we give a definition of oscillations spaces and a characterization of this spaces in terms of box dimension. In Section 5 we give the most remarkable results. In particular, we state there the main theorem of the paper, with the exact values of the maximal and minimal dimensions. Proofs are shifted to Section 6.

I would like to thank Professor António Caetano for the English corrections and clarification of my ideas, graphics, and suggestions for the research paper topic. I specially would like to thank him to make my trip to Jena possible where I had the opportunity to meet Professor Hans Triebel, and to be exposed to a creative and academic environment. In addition, I would to extend my thanks to Professor Triebel for his valuable suggestions concerning selection of the main results, notations and layout of the paper. I truly enjoyed discussing the preliminary versions of the paper with Professor Caetano, and the many discussions that followed based on a good personal and professional relationship, as well as the excellent work environment. His advices greatly contributed for the final quality of this paper. A special thank to Paolo Vettori and Ricardo Pereira for their precious contribution in the creation of the figures included in this paper. 


\section{Besov spaces and class $s *$}

Let us start by recalling in a shortened way the definition of the BesovTriebel-Lizorkin spaces. Afterwards we introduce the concept of class $s *$, the class of the real continuous functions with an integrability $p$ and an "exact" smoothness $s$.

Definition 2.1. (a) Let $x=\left(x_{1}, \ldots, x_{n}\right) \in \mathbb{R}^{n}$. Then $|x|=\sqrt{\sum_{i=1}^{n} x_{i}^{2}}$.

(b) Let $\xi=\left(\xi_{1}, \ldots, \xi_{n}\right) \in \mathbb{R}^{n}$. Then $\xi x=\sum_{i=1}^{n} \xi_{i} x_{i}$.

(c) Let $\alpha=\left(\alpha_{1}, \ldots, \alpha_{n}\right) \in\left(\mathbb{N}_{0}\right)^{n}$. Then $D^{\alpha}$ is the classic derivative operator.

(d) We define the sets $C^{\infty}\left(\mathbb{R}^{n}\right)=\left\{\varphi: \mathbb{R}^{n} \rightarrow \mathbb{C}: D^{\alpha} \varphi\right.$ is continuous for all $\left.\alpha \in\left(\mathbb{N}_{0}\right)^{n}\right\}$ and $C_{0}^{\infty}\left(\mathbb{R}^{n}\right)=\left\{\varphi \in C^{\infty}\left(\mathbb{R}^{n}\right): \varphi\right.$ has a compact support $\}$.

Definition 2.2. Let the class of the Schwartz functions be defined by

$S\left(\mathbb{R}^{n}\right)=\left\{\psi \in C^{\infty}\left(\mathbb{R}^{n}\right)\right.$ with $\left.p_{r, s}(\psi)=\sup _{\mathbb{R}^{n}} \sup _{|\alpha| \leq s}|x|^{r}\left|D^{\alpha} \psi(x)\right|<\infty, \forall r, s \in \mathbb{N}_{0}\right\}$.

The topology of $S\left(\mathbb{R}^{n}\right)$ is defined by this family of semi-norms $p_{r, s}$ with $r, s \in \mathbb{N}_{0}$.

We define also $S^{\prime}\left(\mathbb{R}^{n}\right)$, the dual of $S\left(\mathbb{R}^{n}\right)$ equipped with the weak topology or with the strong topology.

Definition 2.3. We define ([9], p. 13) $F: S\left(\mathbb{R}^{n}\right) \rightarrow S\left(\mathbb{R}^{n}\right)$, the Fourier transform on $S\left(\mathbb{R}^{n}\right)$, by $(F \psi)(\xi)=\frac{1}{(2 \pi)^{\frac{n}{2}}} \int_{\mathbb{R}^{n}} \psi(x) e^{-i \xi x} d x, \forall \xi \in \mathbb{R}^{n}$, where $e$ is the Neper's number. We extend consistently the Fourier transform to $F: S^{\prime}\left(\mathbb{R}^{n}\right) \rightarrow S^{\prime}\left(\mathbb{R}^{n}\right)$ by defining $\langle F T, \psi\rangle=\langle T, F \psi\rangle, \forall \psi \in S\left(\mathbb{R}^{n}\right)$.

Definition 2.4. Let $0<p \leq \infty$. Then we define the Lebesgue spaces

$L_{p}\left(\mathbb{R}^{n}\right)=\left\{f: \mathbb{R}^{n} \rightarrow \mathbb{C}\right.$ measurable with $\left.\|f\|_{L_{p}\left(\mathbb{R}^{n}\right)}=\left(\int_{\mathbb{R}^{n}}|f(t)|^{p} d t\right)^{1 / p}<\infty\right\}$.

(Standard modification for $p=\infty$.)

Definition 2.5. Let $F$ be the Fourier transform on $S\left(\mathbb{R}^{n}\right)$ and $*$ be the convolution operator in $S^{\prime}\left(\mathbb{R}^{n}\right)$.

Let $\varphi \in S\left(\mathbb{R}^{n}\right)$ with $\operatorname{supp}(F \varphi) \subset\left\{\xi \in \mathbb{R}^{n}: 1 / 2 \leq|\xi| \leq 2\right\}$ and $(F \varphi)(\xi)+(F \varphi)\left(2^{-1} \xi\right)=1$ if $1 \leq|\xi| \leq 2$. For all $j \in \mathbb{N}$ we define $\varphi_{j}(x)=2^{j n} \varphi\left(2^{j} x\right), \forall x \in \mathbb{R}^{n}$.

Let $\varphi_{0} \in S\left(\mathbb{R}^{n}\right)$ with $\operatorname{supp}\left(F \varphi_{0}\right) \subset\left\{\xi \in \mathbb{R}^{n}:|\xi| \leq 2\right\}$ and $\left(F \varphi_{0}\right)(\xi)+(F \varphi)\left(2^{-1} \xi\right)=1$ if $|\xi| \leq 2$. 


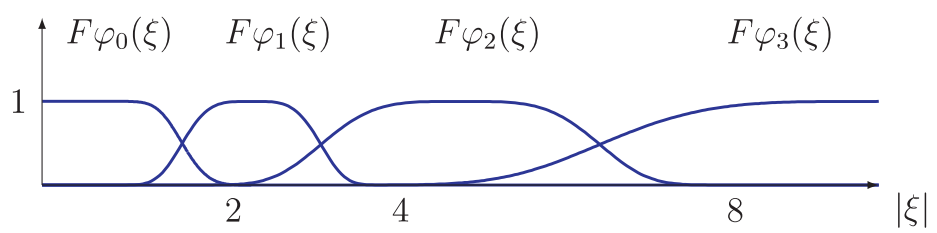

Figure 3. The sequence $\left(F \varphi_{j}\right)_{j \in \mathbb{N}_{0}}$ is a smooth resolution of the unity in frequency, since we have $\sum_{j \geq 0}\left(F \varphi_{j}\right)(\xi)=1, \forall \xi \in \mathbb{R}^{n}$

Consider now $0<p \leq \infty, 0<q \leq \infty$ and $s \in \mathbb{R}$.

We define the Besov spaces

$$
B_{p, q}^{s}\left(\mathbb{R}^{n}\right)=\left\{f \in S^{\prime}\left(\mathbb{R}^{n}\right) \text { with }\|f\|_{B_{p, q}^{s}\left(\mathbb{R}^{n}\right)}=\left(\sum_{j \geq 0}\left(2^{j s}\left\|\varphi_{j} * f\right\|_{L_{p}\left(\mathbb{R}^{n}\right)}\right)^{q}\right)^{1 / q}<\infty\right\} .
$$

(Standard modification if $q=\infty$.)

We define also the Triebel-Lizorkin spaces $F_{p, q}^{s}\left(\mathbb{R}^{n}\right)(0<p<\infty)$ with the quasi-norm $\|f\|_{F_{p, q}^{s}\left(\mathbb{R}^{n}\right)}$, in an analogous way by commuting the order of integration and summation.

Remark. By [9], p. 46 the Besov spaces $B_{p, q}^{s}\left(\mathbb{R}^{n}\right)$ and Triebel-Lizorkin spaces $F_{p, q}^{s}\left(\mathbb{R}^{n}\right)$ are independent (equivalent quasi-norms) of $\varphi$ and $\varphi_{0}$.

We are interested only in functions satisfying the condition (CCS), i.e., real continuous functions with a compact support in $\mathbb{R}^{n}$. By Theorem 1.1, we have the guarantee that the following definition of the class $B_{p}^{s *}\left(\mathbb{R}^{n}\right)$ - or shortly $s *$, the class of the functions with an integrability $p$ and an "exact" smoothness $s$-is non-empty, since that we consider $s>0$.

Definition 2.6. Consider $0<p \leq \infty$ and $s>0$, and suppose that $f$ verify the condition (CCS). Then by definition, $f$ belongs to the set $B_{p}^{s *}\left(\mathbb{R}^{n}\right)$ if and only if for all $\varepsilon>0$ we have

$$
f \in B_{p, p}^{s-\varepsilon}\left(\mathbb{R}^{n}\right) \text { and } f \notin B_{p, p}^{s+\varepsilon}\left(\mathbb{R}^{n}\right) .
$$

We define also the sets $B_{p}^{s-}\left(\mathbb{R}^{n}\right)=\{f$ with (CCS) such that $f \in$ $\left.B_{p, p}^{s-\varepsilon}\left(\mathbb{R}^{n}\right), \forall \varepsilon>0\right\}$ and

$$
B_{p}^{s+}\left(\mathbb{R}^{n}\right)=\left\{f \text { with }(\mathrm{CCS}) \text { such that } f \notin B_{p, p}^{s+\varepsilon}\left(\mathbb{R}^{n}\right), \forall \varepsilon>0\right\} .
$$

Remark. Of course, we have the equality $B_{p}^{s *}\left(\mathbb{R}^{n}\right)=B_{p}^{s-}\left(\mathbb{R}^{n}\right) \cap$ $B_{p}^{s+}\left(\mathbb{R}^{n}\right)$. 


\section{Box dimension}

Next we define upper and lower box dimensions - for more details see [3] - as well the maximal and minimal dimensions.

Definition 3.1. Let $\nu \in \mathbb{N}_{0}$. A dyadic $n$-cube $I$, with volume $|I|=2^{-\nu n}$, is a subset of $\mathbb{R}^{n}$ of the form $I=2^{-\nu}\left([0,1]^{n}+k\right)$, where $\nu \in \mathbb{N}_{0}$ and $k \in \mathbb{Z}^{n}$. (see Figure 4 for the case $n=2$.)

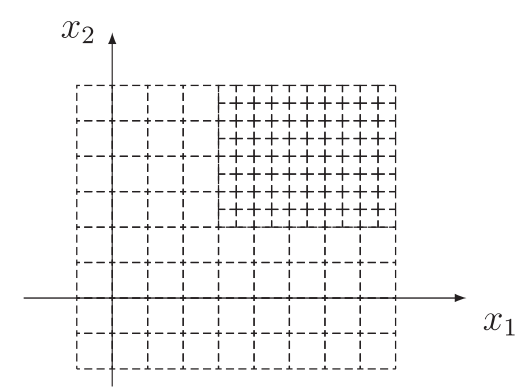

Figure 4. Representation of dyadic squares in the plane $\mathbb{R}^{2}$

Definition 3.2. (a) Let $f$ be a function satisfying the condition (CCS) and let $N(\nu, f)$ be the minimum number of dyadic $(n+1)$-cubes, with volume $|I|=2^{-\nu(n+1)}$, required to cover $\Gamma(f)$ according to Section 1 (see Figure 2 for the case $n=1$ ). Then we define (see [3], pp. 38 and 41 with elementary adaptations) the upper box dimension

$$
\overline{\operatorname{dim}}_{B} \Gamma(f)=\varlimsup_{\nu \rightarrow \infty} \frac{\log _{2} N(\nu, f)}{\nu},
$$

and the lower box dimension $\underline{\operatorname{dim}}_{B} \Gamma(f)=\underline{\lim }_{\nu \rightarrow \infty} \frac{\log _{2} N(\nu, f)}{\nu}$. If these two limits are equal we define the box dimension $\operatorname{dim}_{B} \Gamma(f)$ as the common value of them.

Remark. We can assume $K_{f}=[0,1]^{n}$ - see Section 1 - getting $2^{\nu n} \leq N(\nu, f) \leq 2^{\nu n} 2^{\nu} 2(\sup |f|+1)$ and therefore the inequalities $n \leq$ $\underline{\operatorname{dim}}_{B} \Gamma(f) \leq \overline{\operatorname{dim}}_{B} \Gamma(f) \leq n+1$. Concerning properties about box dimension, see e.g. [3].

Definition 3.3. Let $0<p \leq \infty$, and $s>0$. The classes $B_{p}^{s-}\left(\mathbb{R}^{n}\right)$, $B_{p}^{s+}\left(\mathbb{R}^{n}\right)$ and $B_{p}^{s *}\left(\mathbb{R}^{n}\right)$ were introduced in Definition 2.6. Then we can define the maximal dimension

$$
\overline{\operatorname{Dim}}_{p}^{s}=\sup _{f \in B_{p}^{s-}\left(\mathbb{R}^{n}\right)} \overline{\operatorname{dim}}_{B} \Gamma(f),
$$


and the minimal dimension

$$
\overline{\operatorname{dim}}_{p}^{s}=\inf _{f \in B_{p}^{s+}\left(\mathbb{R}^{n}\right)} \overline{\operatorname{dim}}_{B} \Gamma(f) .
$$

Remark. We have $\overline{\operatorname{dim}}_{p}^{s} \leq \overline{\operatorname{Dim}}_{p}^{s}$ for $0<p \leq \infty$ and $s>0$, because $f_{s} \in B_{p}^{s *}\left(\mathbb{R}^{n}\right)=B_{p}^{s-}\left(\mathbb{R}^{n}\right) \cap B_{p}^{s+}\left(\mathbb{R}^{n}\right)$ with $f_{s}$ as in Theorem 1.1. Furthermore, as we will see these definitions of maximal and minimal dimension coincide with the ones given in the introduction.

\section{Oscillation spaces}

Here we define oscillation spaces and give a characterization of them in terms of upper and lower box dimensions. Later these spaces will be related with Besov spaces.

Definition 4.1. Let $\nu \in \mathbb{N}_{0}$ and $f: \mathbb{R}^{n} \rightarrow \mathbb{R}$ be a continuous function. Then

(a) $\operatorname{osc}_{A}(f)=\sup _{A} f-i n f_{A} f$, if $A$ is a compact of $\mathbb{R}^{n}$ (see Figure 5).

(b) $\operatorname{Osc}(\nu, f)=\sum_{|I|=2^{-\nu n}} \operatorname{osc}_{I}(f)$, where the sum is taken over all dyadic $n$-cubes $I$ as in Definition 3.1.

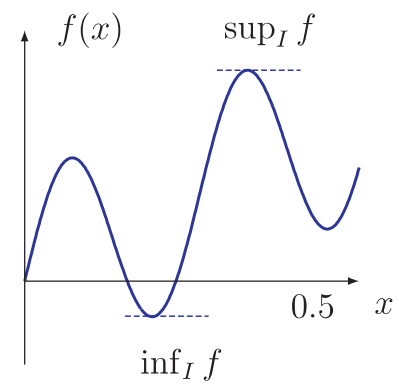

Figure 5. The oscillation of $f$ over $I=[0,0.5]$ is $\operatorname{osc}_{I}(f)=\sup _{I} f-\inf _{I} f$

Definition 4.2. Let $\alpha \in \mathbb{R}$. We define the oscillation spaces

$$
V^{\alpha}\left(\mathbb{R}^{n}\right)=\left\{f: \mathbb{R}^{n} \rightarrow \mathbb{R} \text { continuous with }\|f\|_{V^{\alpha}\left(\mathbb{R}^{n}\right)}<\infty\right\},
$$

where

$$
\|f\|_{V^{\alpha}\left(\mathbb{R}^{n}\right)}=\sup _{\nu \geq 0} 2^{\nu(\alpha-n)} \operatorname{Osc}(\nu, f) .
$$

Consider $\alpha>0$. Analogous to the Definition 2.6, a function $f$ verifying (CCS) belongs to the set $V^{\alpha *}\left(\mathbb{R}^{n}\right)$ if and only if for all $\varepsilon>0$ we have

$$
f \in V^{\alpha-\varepsilon}\left(\mathbb{R}^{n}\right) \text { and } f \notin V^{\alpha+\varepsilon}\left(\mathbb{R}^{n}\right) .
$$


We define also the sets $V^{\alpha-}\left(\mathbb{R}^{n}\right)=\{f$ with (CCS) such that $f \in$ $\left.V^{\alpha-\varepsilon}\left(\mathbb{R}^{n}\right), \forall \varepsilon>0\right\}$ and $V^{\alpha+}\left(\mathbb{R}^{n}\right)=\{f$ with (CCS) such that $f \notin$ $\left.V^{\alpha+\varepsilon}\left(\mathbb{R}^{n}\right), \forall \varepsilon>0\right\}$.

\section{Remark.}

(a) Let $\alpha \in \mathbb{R}$. Then $\|\cdot\|_{V^{\alpha}\left(\mathbb{R}^{n}\right)}$ is a semi-norm in the space $V^{\alpha}\left(\mathbb{R}^{n}\right)$.

(b) Let $\alpha \geq 0$ and $f \in C^{\alpha}\left(\mathbb{R}^{n}\right)$ satisfying (CCS). Then $f \in V^{\alpha}\left(\mathbb{R}^{n}\right)$.

(c) Of course, we have the equality $V^{\alpha *}\left(\mathbb{R}^{n}\right)=V^{\alpha-}\left(\mathbb{R}^{n}\right) \cap V^{\alpha+}\left(\mathbb{R}^{n}\right)$.

Definition 4.3. Let $0<p \leq \infty, s \in \mathbb{R}$ and $\alpha \in \mathbb{R}$. Then, we define the sets $\underline{B}_{p, \infty}^{s}\left(\mathbb{R}^{n}\right)=\left\{f \in S^{\prime}\left(\mathbb{R}^{n}\right)\right.$ with $\|f\|_{\underline{B}_{p, \infty}^{s}\left(\mathbb{R}^{n}\right)}=\underline{\lim }_{j \rightarrow \infty} 2^{j s} \| \varphi_{j} *$ $\left.f \|_{L_{p}\left(\mathbb{R}^{n}\right)}<\infty\right\}$ and $\underline{V}^{\alpha}\left(\mathbb{R}^{n}\right)=\left\{f: \mathbb{R}^{n} \rightarrow \mathbb{R}\right.$ continuous with $\|f\|_{\underline{V}^{\alpha}\left(\mathbb{R}^{n}\right)}=$ $\left.\varliminf_{\nu \rightarrow \infty} 2^{\nu(\alpha-n)} O \operatorname{sc}(\nu, f)<\infty\right\}$.

In an analogous way as in Definition 4.2 , if $\alpha>0$ we define the sets $\underline{V}^{\alpha *}\left(\mathbb{R}^{n}\right), \underline{V}^{\alpha-}\left(\mathbb{R}^{n}\right)$ and $\underline{V}^{\alpha+}\left(\mathbb{R}^{n}\right)$, and similarly the sets $\underline{B}_{p}^{s *}\left(\mathbb{R}^{n}\right)$, $\underline{B}_{p}^{s-}\left(\mathbb{R}^{n}\right)$ and $\underline{B}_{p}^{s+}\left(\mathbb{R}^{n}\right)$ if $s>0$.

Theorem 4.1. Let $f$ be a function satisfying the condition (CCS). Then we have the equivalences

$$
\overline{\operatorname{dim}}_{B} \Gamma(f) \leq n+1-\gamma \Longleftrightarrow f \in V^{\gamma-}\left(\mathbb{R}^{n}\right), \text { if } 0<\gamma \leq 1
$$

and

$$
\overline{\operatorname{dim}}_{B} \Gamma(f) \geq n+1-\gamma \Longleftrightarrow f \in V^{\gamma+}\left(\mathbb{R}^{n}\right), \text { if } 0 \leq \gamma<1 .
$$

Theorem 4.2. Let $f$ be a function satisfying the condition (CCS), and $\eta: \mathbb{R}^{n} \rightarrow \mathbb{R}$ such that $\eta \in C^{1}\left(\mathbb{R}^{n}\right)$. Then we have

$$
\overline{\operatorname{dim}}_{B} \Gamma(\eta f) \leq \overline{\operatorname{dim}}_{B} \Gamma(f) .
$$

Remark 4.3. With the same arguments we can prove that Lemma 6.1, and therefore Theorems 4.1 and 4.2 , are also true for the lower counterparts $\underline{\operatorname{dim}}_{B}$ and $\underline{V}$ - see Definitions 3.2 and 4.3 .

\section{Main results}

All unimportant positive constants are denoted by $c$. When in the same expression they are distinguished by $c, c^{\prime}, c^{\prime \prime}, \ldots$ Sometimes these constants have an index, for example $c_{m}$ is a constant that depends on $m$. Furthermore, [.] represents the integer part of a non-negative number. In this Section we develop the main results, namely embeddings and inclusions between the oscillation spaces $V^{\alpha}\left(\mathbb{R}^{n}\right)$ and the Besov spaces $B_{p, q}^{s}\left(\mathbb{R}^{n}\right)$. In papers [4] and [5] we find very fine embeddings that relate oscillation spaces and Besov spaces, nevertheless those results are somewhat complementary 
to the ones we need here. We state also here the main theorem of the paper, that gives the exact values for the maximal and minimal upper box dimensions. Maximal lower box dimension is also given. For more details concerning results and proofs, including counterexamples for situations to which the results cannot be extended, see [1].

Theorem 5.1. Let $0<p<\infty$ and $\gamma \geq \frac{n}{p}$. Let $m \in \mathbb{N}$ and $f: \mathbb{R}^{n} \rightarrow \mathbb{R}$ with $f \in B_{p, 1}^{\gamma}\left(\mathbb{R}^{n}\right)$ and supp $f \subset[-m, m]^{n}$. Then we have

$$
\|f\|_{V_{\min (1, \gamma)\left(\mathbb{R}^{n}\right)}} \leq c_{m}\|f\|_{B_{p, 1}^{\gamma}\left(\mathbb{R}^{n}\right)} .
$$

Corollary 5.2. Let $0<p \leq \infty$ and $\gamma>\frac{n}{p}$. Let $f \in B_{p}^{\gamma-}\left(\mathbb{R}^{n}\right)$. Then we have (see Figure 6)

$$
\overline{\operatorname{dim}}_{B} \Gamma(f) \leq n+1-\min (1, \gamma)
$$

In [8], p. 21 we find a result similar to the Corollary 5.2. For a comparison between [8], p. 21 and the above Corollary see [9], pp. 189 and 192 -also Lemma 6.5 concerning the case $0<p<1$. In [10], p. 120 we find work concerning the case $p=\infty$. With a proof similar to the one of the Theorem 5.1 , we get also the embedding $B_{1,1}^{n}\left(\mathbb{R}^{n}\right) \hookrightarrow V^{1}\left(\mathbb{R}^{n}\right)$.

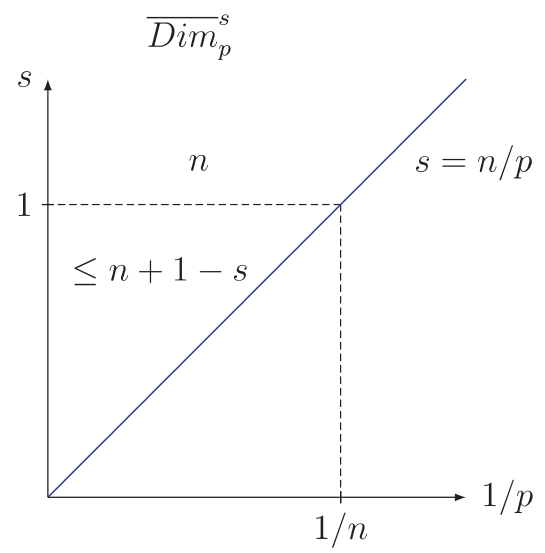

Figure 6. The Corollary 5.2 gives $\overline{\operatorname{Dim}}_{p}^{s} \leq n+1-\min (1, s)$ for $0<p \leq \infty$ and $s>\frac{n}{p}$ 
Theorem 5.3. Let $0<p<\infty$ and $\gamma^{\prime}>\gamma$. Suppose that $f \in$ $V^{\gamma^{\prime} \max (1, p)}\left(\mathbb{R}^{n}\right)$ has a compact support. Then we have

$$
f \in B_{p, \infty}^{\gamma}\left(\mathbb{R}^{n}\right) .
$$

Corollary 5.4. Let $0<p<\infty$ and $0 \leq \gamma<\frac{1}{\max (1, p)}$. Let $f \in$ $B_{p}^{\gamma+}\left(\mathbb{R}^{n}\right)$. Then by Theorems 5.3 and 4.1 we have (see Figure 7)

$$
\overline{\operatorname{dim}}_{B} \Gamma(f) \geq n+1-\gamma \max (1, p) .
$$

Remark 5.5. (a) With the same proof we can show that the Theorem 5.3 is true also for $\underline{V}$ and $\underline{B}$ in place of the standard counterparts. Hence by Remark 4.3, we have that the Corollary 5.4 is valid also for the lower counterparts $\underline{B}$ and $\underline{\operatorname{dim}}_{B}$.

(b) With a similar proof to the one of the Theorem 5.3, we get the embedding $\left(V^{\gamma} \cap L_{1}\right)\left(\mathbb{R}^{n}\right) \hookrightarrow B_{1, \infty}^{\gamma}\left(\mathbb{R}^{n}\right)$ for $\gamma \in \mathbb{R}$, where $\|$. $\left\|_{\left(V^{\gamma} \cap L_{1}\right)\left(\mathbb{R}^{n}\right)}=\right\| \cdot\left\|_{V^{\gamma}\left(\mathbb{R}^{n}\right)}+\right\| \cdot \|_{L_{1}\left(\mathbb{R}^{n}\right)}$.

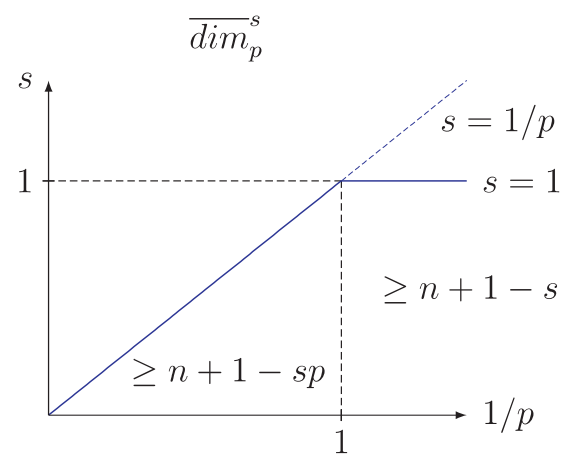

Figure 7. The Corollary 5.4 gives $\overline{\operatorname{dim}}_{p}^{s} \geq n+1-s \max (1, p)$ for

$$
0<p<\infty \text { and } 0<s<\frac{1}{\max (1, p)}
$$

As we can see, the Corollary 5.4 generalizes the Theorem 1.2 given in Section 1. Next we will construct extreme functions with the objective of reaching the maximal and minimal dimensions. In Definition 5.1 we construct a function $\Theta_{0}$ that materialize the maximal dimension for $0<$ $p<\infty$ and $0<s \leq \frac{n}{p}$, given in Theorem 5.6. In Definition 5.2 we have a more refined function in order to get the Theorem 5.8, which strengthens the Theorem 5.6.

Definition 5.1. Let $\psi: \mathbb{R}^{n} \rightarrow \mathbb{R}$ with $\psi \in S\left(\mathbb{R}^{n}\right), \psi(0, \ldots, 0) \neq 0$ and $\operatorname{supp}(F \psi) \subset\left\{\xi \in \mathbb{R}^{n}: 1-a \leq|\xi| \leq 1+a\right\}$ for $a>0$ sufficiently small. 
Let $j_{0} \in \mathbb{N}$ sufficiently large.

(I) For $n=1$ : For each $j \in \mathbb{N} \backslash\{1,2\}$ let $\Theta_{j}: \mathbb{R} \rightarrow \mathbb{R}$ with

$$
\Theta_{j}=\psi\left(2^{j+j_{0}}\left(\cdot-\frac{1}{\operatorname{lnj}}\right)\right) .
$$

Let $\Theta: \mathbb{R} \rightarrow \mathbb{R}$ with $\Theta=\sum_{j \geq 3} \Theta_{j}$. (Pointwise convergence and also convergence in $S^{\prime}(\mathbb{R})$ with the weak topology to the same function.)

Let $\phi: \mathbb{R} \rightarrow \mathbb{R}$ with $\phi \in C_{0}^{\infty}(\mathbb{R})$ and with $\phi(x)=x, \forall x \in[0,1]$.

Let $\Theta_{0}: \mathbb{R} \rightarrow \mathbb{R}$ with $\Theta_{0}=\phi \Theta$.

(II) For $n \in \mathbb{N} \backslash\{1\}$ : For each $j \in \mathbb{N} \backslash\{1,2\}$ and for $l \in\left\{1, \ldots, j^{2(n-1)}\right\}$ let $\Theta_{j, l}: \mathbb{R}^{n} \rightarrow \mathbb{R}$ with

$$
\Theta_{j, l}=\psi\left(2^{j+j_{0}}(\cdot-m(j, l))\right),
$$

and with $m(j, l)=\left(\frac{1}{\ln j}, \frac{s_{2, l}}{j^{2}}, \ldots, \frac{s_{n, l}}{j^{2}}\right)$, where the $j^{2(n-1)}$ different values of $l$ correspond to the different values of $\left(s_{2, l}, \ldots, s_{n, l}\right) \in\left\{1, \ldots, j^{2}\right\}^{n-1}$.

Let $\Theta: \mathbb{R}^{n} \rightarrow \mathbb{R}$ with $\Theta=\sum_{j \geq 3} \sum_{l=1}^{j^{2(n-1)}} \Theta_{j, l}$. (Pointwise convergence and also convergence in $S^{\prime}\left(\mathbb{R}^{n}\right)$ with the weak topology to the same function.)

Let $\phi: \mathbb{R}^{n} \rightarrow \mathbb{R}$ with $\phi \in C_{0}^{\infty}\left(\mathbb{R}^{n}\right)$ and with $\phi(x)=\phi\left(x_{1}, \ldots, x_{n}\right)=$ $x_{1}, \forall x \in[0,1]^{n}$.

Let $\Theta_{0}: \mathbb{R}^{n} \rightarrow \mathbb{R}$ with $\Theta_{0}=\phi \Theta$.

Theorem 5.6. Let $\Theta_{0}$ as in Definition 5.1. Then (see Figure 8)

(a) $\Theta_{0}$ is continuous,

(b) $\operatorname{dim}_{B} \Gamma\left(\Theta_{0}\right)=n+1$,

and

(c) $\Theta_{0} \in B_{p}^{\frac{n}{p}-}\left(\mathbb{R}^{n}\right)$ for $0<p \leq \infty$.

As mentioned in the Introduction, the Theorem 1.2 cannot be improved with the inequality $\leq$. In the same way, only the part $\geq$ of the claimed Theorem 4.2 of [2], p. 220 is true. This follows by the Theorem 5.6 (or Theorem 5.8). The failure of part $\leq$ of this claim in [2], p. 220 comes from the false inequality (4.3) of [2], p. 219, which originates the false inequality (4.1) of [2], p. 219. We can see in Remark 5.7 that (4.1) $\|f\|_{V^{\gamma}(\mathbb{R})} \leq c\|f\|_{B_{1,1}^{\gamma}(\mathbb{R})}$ with $0<\gamma<1$ of [2], p. 219 is false, even with the restriction of $f$ to the real functions of the $C_{0}^{\infty}(\mathbb{R})$. Kamont and Wolnik in [6] constructed another counterexample for this claim of [2], p. 220. 


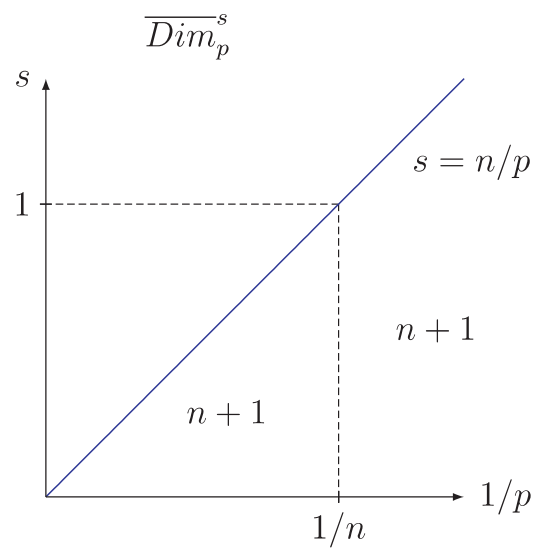

Figure 8. The Theorem 5.6 gives $\overline{\operatorname{Dim}}_{p}^{s}=n+1$ for $0<p<\infty$ and $0<s \leq \frac{n}{p}$

Remark 5.7. Let $\varphi$ be as in Definition 2.5, and with $\operatorname{supp}(F \varphi) \subset\{\xi \in$ $\left.\mathbb{R}^{n}: \frac{1}{2}(1+a) \leq|\xi| \leq 2(1-a)\right\}$ and $(F \varphi)(\xi)=1$ if $1-a \leq|\xi| \leq 1+a$, for some $a>0$.

Let $\psi: \mathbb{R}^{n} \rightarrow \mathbb{R}$ with $\psi \in S\left(\mathbb{R}^{n}\right) \backslash\{0\}$ and $\operatorname{supp}(F \psi) \subset\{\xi \in \mathbb{R}: 1-a \leq$ $|\xi| \leq 1+a\}$.

Furthermore, let $\phi: \mathbb{R}^{n} \rightarrow \mathbb{R}$ with $\phi \in C_{0}^{\infty}\left(\mathbb{R}^{n}\right)$ and suppose that $(\phi \psi)(0, \ldots, 0) \neq 0$.

For each $j \in \mathbb{N}$ let $\psi_{j}=\psi\left(2^{j}\right.$. $)$ and $\phi_{j}=\phi \psi_{j}$.

Then for $\alpha \in \mathbb{R}$ we have $\left\|\phi_{j}\right\|_{V^{\alpha}\left(\mathbb{R}^{n}\right)} \geq\left\|\phi_{j}\right\|_{L_{\infty}\left(\mathbb{R}^{n}\right)} \geq c>0$.

Nevertheless, from the property $F(\Phi * \Psi)=(2 \pi)^{\frac{n}{2}}(F \Phi)(F \Psi)$ in $S^{\prime}\left(\mathbb{R}^{n}\right)$ we have $\varphi_{j} * \psi_{j}=(2 \pi)^{\frac{n}{2}} \psi_{j}$ and then for $0<p \leq \infty, 0<q \leq \infty$ and $s \in \mathbb{R}$ we have $\left\|\psi_{j}\right\|_{B_{p, q}^{s}\left(\mathbb{R}^{n}\right)}=c 2^{j\left(s-\frac{n}{p}\right)}$. Hence by the Lemma 6.6 we have

$$
\left\|\phi_{j}\right\|_{B_{p, q}^{s}\left(\mathbb{R}^{n}\right)} \leq c 2^{j\left(s-\frac{n}{p}\right)} .
$$

Definition 5.2. Let $\psi: \mathbb{R}^{n} \rightarrow \mathbb{R}$ with $\psi \in S\left(\mathbb{R}^{n}\right) \backslash\{0\}$ and $\operatorname{supp}(F \psi) \subset$ $\left\{\xi \in \mathbb{R}^{n}: 1-a \leq|\xi| \leq 1+a\right\}$ for $a>0$ sufficiently small.

Let $0<\lambda \leq 1$. For each $j \in \mathbb{N} \backslash\{1,2\}$ let $k(j)=\left[2^{j(1-\lambda)}\right]$.

(I) For $n=1$ : For each $j \in \mathbb{N} \backslash\{1,2\}$ and for $k \in\{1, \ldots, k(j)\}$ let $m(j, k)=\frac{1}{\ln j}-k 2^{-j}$ and $\Theta_{j, k}: \mathbb{R} \rightarrow \mathbb{R}$ with

$$
\Theta_{j, k}=\psi\left(2^{j}(\cdot-m(j, k))\right) .
$$

Let $\Theta: \mathbb{R} \rightarrow \mathbb{R}$ with $\Theta=\sum_{j \geq 3} \sum_{k=1}^{k(j)} \Theta_{j, k}$. (Pointwise convergence and also convergence in $S^{\prime}(\mathbb{R})$ with the weak topology to the same function.) 
Let $\phi: \mathbb{R} \rightarrow \mathbb{R}$ with $\phi \in C_{0}^{\infty}(\mathbb{R})$ and with $\phi(x)=x, \forall x \in[0,1]$.

Let $\Theta_{0}: \mathbb{R} \rightarrow \mathbb{R}$ with $\Theta_{0}=\phi \Theta$.

(II) For $n \in \mathbb{N} \backslash\{1\}$ : Let $0 \leq h \leq n-1$. For each $j \in \mathbb{N} \backslash\{1,2\}$ let $d(j)=\left[2^{j \frac{n-1-h}{n-1}}\right] .\left(\right.$ Modification $d(j)=j^{2}$ if $h=n-1$.)

For each $j \in \mathbb{N} \backslash\{1,2\}$, for $k \in\{1, \ldots, k(j)\}$ and for $l \in\left\{1, \ldots, d(j)^{n-1}\right\}$ let $m(j, k, l)=\left(\frac{1}{\ln j}-k 2^{-j}, \frac{s_{2, l}}{d(j)}, \ldots, \frac{s_{n, l}}{d(j)}\right)$, where for each fixed $(j, k)$ the $d(j)^{n-1}$ different values of $l$ correspond to the different values of $\left(s_{2, l}, \ldots, s_{n, l}\right) \in\{1, \ldots, d(j)\}^{n-1}$.

Let also $\Theta_{j, k, l}: \mathbb{R} \rightarrow \mathbb{R}$ with

$$
\Theta_{j, k, l}=\psi\left(2^{j}(\cdot-m(j, k, l))\right) .
$$

Let $\Theta: \mathbb{R}^{n} \rightarrow \mathbb{R}$ with $\Theta=\sum_{j \geq 3} \sum_{k=1}^{k(j)} \sum_{l=1}^{d(j)^{n-1}} \Theta_{j, k, l}$. (Pointwise convergence and also convergence in $S^{\prime}\left(\mathbb{R}^{n}\right)$ with the weak topology to the same function.)

Let $\phi: \mathbb{R}^{n} \rightarrow \mathbb{R}$ with $\phi \in C_{0}^{\infty}\left(\mathbb{R}^{n}\right)$ and with $\phi(x)=\phi\left(x_{1}, \ldots, x_{n}\right)=$ $x_{1}, \forall x \in[0,1]^{n}$.

Let $\Theta_{0}: \mathbb{R}^{n} \rightarrow \mathbb{R}$ with $\Theta_{0}=\phi \Theta$.

Theorem 5.8. Let $0 \leq h \leq n-1,0<\lambda \leq 1$ and $\Theta_{0}$ as in Definition 5.2. Then

(a) $\Theta_{0}$ is continuous,

(b) $\operatorname{dim}_{B} \Gamma\left(\Theta_{0}\right)=n+1$, and

(c) $\Theta_{0} \in B_{p}^{\frac{h+\lambda}{p}} *\left(\mathbb{R}^{n}\right)$ for $0<p \leq \infty$.

The Theorem 5.8 strengthens the Theorem 5.6. In fact, if in Definition 5.2 we additionally impose $\psi(0, \ldots, 0) \neq 0$, replace $\psi$ by $\psi\left(2^{j_{0}}\right.$. $)$ with $j_{0} \in \mathbb{N}$ large, take $h=n-1, \lambda=1$ and simplify $\frac{1}{\ln j}-2^{-j}$ by $\frac{1}{\ln j}$, then we get the same $\Theta$ and $\Theta_{0}$ of the Definition 5.1.

In the following Definition 5.3, the function $\Lambda_{0}$ materializes the minimal dimension for $1 \leq p<\infty$ and $0<s \leq \frac{1}{p}$, given in Theorem 5.9. We can realize that the representation in wavelets, or even in quarks in the context of [11], is an excellent alternative to Definitions 5.1, 5.2 and 5.3 in order to construct extremal functions. Such approach is given in [5], dealing with a multi-fractal analysis in wavelet expansions.

Definition 5.3. Let $\psi: \mathbb{R}^{n} \rightarrow \mathbb{R}$ with $\psi \in S\left(\mathbb{R}^{n}\right) \backslash\{0\}$ and $\operatorname{supp}(F \psi) \subset$ $\left\{\xi \in \mathbb{R}^{n}: 1-a \leq|\xi| \leq 1+a\right\}$ for $a>0$ sufficiently small.

Let $0<\lambda \leq 1$. For each $j \in \mathbb{N}$ let $k(j)=\left[\left(1-2^{-\lambda}\right) 2^{j(1-\lambda)}\right]$. 
In what follows, for $\lambda=1$ we modify $k(j)=1$ and replace $2^{-j \lambda}-k 2^{-j}$ by $2^{-j}$ and $\frac{1}{j}$ by $\frac{1}{j^{2}}$.

(I) For $n=1$ : For each $j \in \mathbb{N}$ and for $k \in\{1, \ldots, k(j)\}$ let $m(j, k)=$ $2^{-j \lambda}-k 2^{-j}$ and $\Lambda_{j, k}: \mathbb{R} \rightarrow \mathbb{R}$ with

$$
\Lambda_{j, k}=\frac{1}{j} \psi\left(2^{j}(\cdot-m(j, k))\right) .
$$

Let $\Lambda: \mathbb{R} \rightarrow \mathbb{R}$ with $\Lambda=\sum_{j \geq 1} \sum_{k=1}^{k(j)} \Lambda_{j, k}$. (Pointwise convergence and also convergence in $S^{\prime}(\mathbb{R})$ with the weak topology to the same function.)

Let $\phi: \mathbb{R} \rightarrow \mathbb{R}$ with $\phi \in C_{0}^{\infty}(\mathbb{R})$ and with $\phi(x)=1, \forall x \in[0,1]$.

Let $\Lambda_{0}: \mathbb{R} \rightarrow \mathbb{R}$ with $\Lambda_{0}=\phi \Lambda$.

(II) For $n \in \mathbb{N} \backslash\{1\}$ : For each $j \in \mathbb{N}$, for $k \in\{1, \ldots, k(j)\}$ and for $l \in\left\{1, \ldots, 2^{j(n-1)}\right\}$ let $m(j, k, l)=\left(2^{-j \lambda}-k 2^{-j}, \frac{s_{2, l}}{2^{j}}, \ldots, \frac{s_{n, l}}{2^{j}}\right)$, where for each fixed $(j, k)$ the $2^{j(n-1)}$ different values of $l$ correspond to the different values of $\left(s_{2, l}, \ldots, s_{n, l}\right) \in\left\{1, \ldots, 2^{j}\right\}^{n-1}$.

Let also $\Lambda_{j, k, l}: \mathbb{R} \rightarrow \mathbb{R}$ with

$$
\Lambda_{j, k, l}=\frac{1}{j} \psi\left(2^{j}(\cdot-m(j, k, l))\right) .
$$

Let $\Lambda: \mathbb{R}^{n} \rightarrow \mathbb{R}$ with $\Lambda=\sum_{j \geq 1} \sum_{k=1}^{k(j)} \sum_{l=1}^{2^{j(n-1)}} \Lambda_{j, k, l}$. (Pointwise convergence and also convergence in $S^{\prime}\left(\mathbb{R}^{n}\right)$ with the weak topology to the same function.)

Let $\phi: \mathbb{R}^{n} \rightarrow \mathbb{R}$ with $\phi \in C_{0}^{\infty}\left(\mathbb{R}^{n}\right)$ and with $\phi(x)=1, \forall x \in[0,1]^{n}$.

Let $\Lambda_{0}: \mathbb{R}^{n} \rightarrow \mathbb{R}$ with $\Lambda_{0}=\phi \Lambda$.

Theorem 5.9. Let $0<\lambda \leq 1$ and $\Lambda_{0}$ as in Definition 5.3. Then

(a) $\Lambda_{0}$ is continuous,

(b) $\operatorname{dim}_{B} \Gamma\left(\Lambda_{0}\right)=n+1-\lambda$, and

(c) $\Lambda_{0} \in B_{p}^{\frac{\lambda}{p} *}\left(\mathbb{R}^{n}\right)$ for $0<p \leq \infty$.

At this moment we are in a position to summarize the obtained results, in terms of maximal and minimal dimensions. The following Theorem is the main theorem of this paper and makes exactly this.

Theorem 5.10 (Main Theorem). Let $0<p \leq \infty$ and $s>0$. Then we have (see Figures 9, 10 and 11)

(a) $\overline{\operatorname{Dim}}_{p}^{s}=n+1$ if $0<s \leq \frac{n}{p}$,

(b) $\overline{D i m}_{p}^{s}=n+1-\min (1, s)$ if $s>\frac{n}{p}$, and 
(c) $\overline{\operatorname{dim}}_{p}^{s}=n+1-s \max (1, p)$ if $0<s \leq \frac{1}{\max (1, p)}$,

(d) $\overline{\operatorname{dim}}_{p}^{s}=n$ if $s \geq \frac{1}{\max (1, p)}$.

Proof. In Theorem 5.6 we proved (a). The argument in [13], p. 202-203 proves Theorem 1.1 and also the relation $f_{s} \in \underline{B}_{p}^{s *}\left(\mathbb{R}^{n}\right)$ for $0<p \leq \infty$ and $s>0$. Hence, by Corollary 5.2 with $\frac{n}{\gamma}+1$ in place of $p$, and by Remark 5.5(a) with 1 in place of $p$ we have the equality $\operatorname{dim}_{B} \Gamma\left(f_{s}\right)=n+1-\min (1, s)$ for $s>0$. Hence we have $\overline{\operatorname{dim}}_{p}^{s} \leq n+1-\min (1, s) \leq \overline{\operatorname{Dim}}_{p}^{s}$ for $0<p \leq \infty$ and $s>0$. By Corollary 5.2 we have also (b). It remains us to prove (c) and $(\mathrm{d})$.

(i) Let $0<p<\infty$. By Corollary 5.4 and remark of Definition 3.2 we have $\overline{\operatorname{dim}}_{p}^{s} \geq n+1-s \max (1, p)$ for $0<s \leq \frac{1}{\max (1, p)}$. On the other hand the Theorem 5.9 gives $\overline{\operatorname{dim}}_{p}^{s} \leq n+1-s p$ for $0<s \leq \frac{1}{p}$. Hence we have (c).

(ii) Let $s>\frac{1}{p}$, and let $\Lambda_{0}$ as in Definition 5.3 if we take $\lambda=1$ and multiply $\Lambda_{j, k}$ and $\Lambda_{j, k, l}$ by the factor $2^{j\left(\frac{1}{p}-s\right)}$. Then, of course $\Lambda_{0}$ is continuous, and by the same proof of Theorem 5.9-(c) we obtain $\Lambda_{0} \in B_{p}^{s *}\left(\mathbb{R}^{n}\right)$. By calculations like in the estimation of $\sum_{j}^{\prime \prime \prime *}$ in part (ii3) of the proof of Theorem 5.9-(b) we obtain $\operatorname{dim}_{B} \Gamma\left(\Lambda_{0}\right)=n$. Hence we have (d).

Remark 5.11. (a) As we can see in arguments in the proof of Theorem 5.10, the supremum $\overline{\operatorname{Dim}}_{p}^{s}$ is a maximum and the infimum $\overline{\operatorname{dim}}_{p}^{s}$ is a minimum.

(b) If for taking the supremum and the infimum in Definition 3.3 we suppose $f \in B_{p}^{s *}\left(\mathbb{R}^{n}\right)$, then we have the same results of the Theorem 5.10 for $\overline{\operatorname{Dim}}_{p}^{s}$ and $\overline{\operatorname{dim}}_{p}^{s}$, including part (a) of present remark. In fact, as we can see in the proof of Theorem, we need only replace the Theorem 5.6 by the more general Theorem 5.8.

(c) We define $\underline{\operatorname{Dim}}_{p}^{s}$ if for taking the supremum in Definition 3.3 we replace $\overline{\operatorname{dim}}_{B}$ by $\underline{\operatorname{dim}}_{B}$. As can be seen in the proof of Theorem 5.10 , we have $\underline{\operatorname{Dim}}_{p}^{s}=\overline{\operatorname{Dim}}_{p}^{s}$ for $0<p \leq \infty$ and $s>0$. We have also the counterpart for parts (a) and (b) of present remark.

(d) Although not proved in this paper, if in Definition 3.3 we relax the continuity in a subset of $\mathbb{R}^{n}$ of upper box dimension $n-1$ (for example a hyperplane), then we have the same results of the Theorem 5.10 for $\overline{\operatorname{Dim}}_{p}^{s}$ and $\overline{\operatorname{dim}}_{p}^{s}$, including the remaining previous parts of the present remark. 


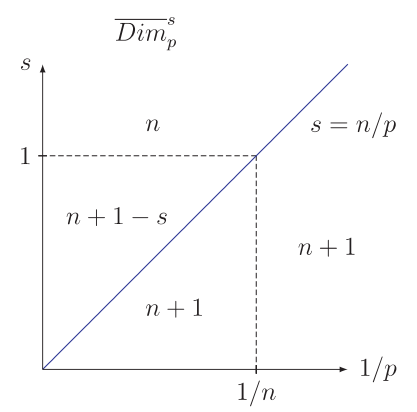

Figure 9. The Theorem 5.10 gives the exact values of $\overline{\operatorname{Dim}}_{p}^{s}$ for $0<p \leq \infty$ and $s>0$

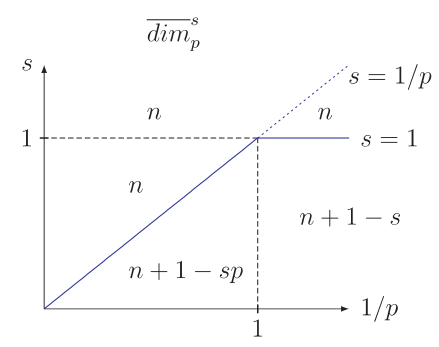

Figure 10. The Theorem 5.10 gives the exact values of $\overline{\operatorname{dim}}_{p}^{s}$ for $0<p \leq \infty$ and $s>0$

We also present an alternative graphical representation, to facilitate the interpretation (see Figure 11).

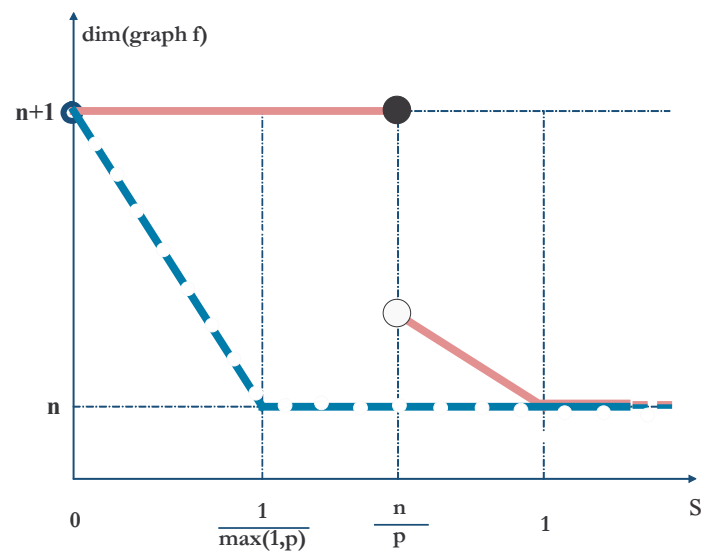

Figure 11. Case $\frac{n}{p}<1$. The solid line represents $\overline{\operatorname{Dim}}_{p}^{s}$ and the dashed line represents $\overline{\operatorname{dim}}_{p}^{s}$. For the case $\frac{n}{p}>1$ the solid line is simpler 


\section{Proofs}

Many of the proofs are outlined in order to save space and to focus in the main ideas. For details about results and proofs see [1]. In this reference we find also counterexamples for situations to which we cannot extend the results presented here.

Definition 6.1. Let $\nu \in \mathbb{N}_{0}$ and $f$ be a continuous function. Then we define $\varphi(\nu, f)=\frac{O s c(\nu, f)}{2^{\nu(n-1)}}$ and $\varphi^{+}(\nu, f)=\max \{\varphi(\nu, f), 1\}$.

Lemma 6.1. Let $f$ be a function satisfying the condition (CCS). Then we have $\overline{\operatorname{dim}}_{B} \Gamma(f)=n+\varlimsup_{\lim _{\nu \rightarrow \infty}} \frac{\log _{2} \varphi^{+}(\nu, f)}{\nu}$. If $\varphi$ is greater or equal to

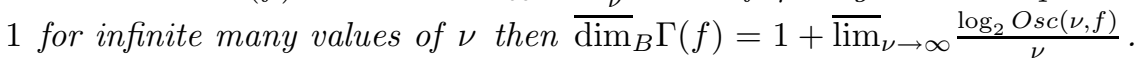
(respectively $\geq$ for the general case.)

Proof of Lemma 6.1. We can assume $K_{f}=[0,1]^{n}$ - see Section 1. Let $N(\nu, I, f)$ be the minimum number of dyadic $(\mathrm{n}+1)$-cubes required to cover the graph of $\left.f\right|_{I}$, where $I \subset[0,1]^{n}$ is a $n$-cube with volume $|I|=2^{-\nu n}$, as in Definition 3.1. Then we have $2^{-\nu}(N(\nu, I, f)-2)<\operatorname{osc}_{I}(f) \leq 2^{-\nu} N(\nu, I, f)$.

By summing over all $I \subset[0,1]^{n}$ we get $2^{-\nu} N(\nu, f)-2^{\nu(n-1)} 2<$ $O s c(\nu, f) \leq 2^{-\nu} N(\nu, f)$, and by multiplying by $2^{-\nu(n-1)}$ we obtain $2^{-\nu n} N(\nu, f)-2<\varphi(\nu, f) \leq 2^{-\nu n} N(\nu, f)$. Of course we have also $N(\nu, f) \geq 2^{\nu n}$, and therefore $2^{\nu n} \varphi^{+}(\nu, f) \leq N(\nu, f)<2^{\nu n}(\varphi(\nu, f)+2)$. Hence we obtain

$$
\begin{aligned}
n+\frac{\log _{2} \varphi^{+}(\nu, f)}{\nu} & \leq \frac{\log _{2} N(\nu, f)}{\nu} \\
& <n+\frac{\log _{2}(\varphi(\nu, f)+2)}{\nu} \\
& \leq n+\frac{\log _{2}\left(3 \varphi^{+}(\nu, f)\right)}{\nu} .
\end{aligned}
$$

Proof of Theorem 4.1. We prove two of the four implications, since the other two follow immediately.

(i) We use the inequality of Lemma 6.1. Let $0<\gamma \leq 1$ and $\overline{\operatorname{dim}}_{B} \Gamma(f) \leq$ $n+1-\gamma$.

For every $\varepsilon>0, \exists \nu_{\varepsilon} \in \mathbb{N}_{0}$ such that $\frac{\log _{2} \operatorname{Osc}(\nu, f)}{\nu} \leq n-\gamma+\varepsilon, \forall \nu \geq \nu_{\varepsilon}$. So $O s c(\nu, f) \leq 2^{\nu(n-\gamma+\varepsilon)}, \forall \nu \geq \nu_{\varepsilon}$ and then we have $f \in V^{\gamma-\varepsilon}\left(\mathbb{R}^{n}\right)$. Hence

$$
f \in V^{\gamma-}\left(\mathbb{R}^{n}\right) .
$$

(ii) We use the second equality of Lemma 6.1. Let $0 \leq \gamma<1$ and $\overline{\operatorname{dim}}_{B} \Gamma(f) \geq n+1-\gamma$. 
Then $\exists\left(k_{\nu}\right)_{\nu \in \mathbb{N}} \subset \mathbb{N}$ with $\lim _{\nu \rightarrow \infty} k_{\nu}=\infty$ such that $\lim _{\nu \rightarrow \infty} \frac{\log _{2} O s c\left(k_{\nu}, f\right)}{k_{\nu}} \geq n-\gamma$.

For every $\varepsilon>0, \exists \nu_{\varepsilon} \in \mathbb{N}_{0}$ such that $\frac{\log _{2} O s c\left(k_{\nu}, f\right)}{k_{\nu}} \geq n-\gamma-\varepsilon, \forall \nu \geq \nu_{\varepsilon}$. So $\operatorname{Osc}\left(k_{\nu}, f\right) \geq 2^{k_{\nu}(n-\gamma-\varepsilon)}, \forall \nu \geq \nu_{\varepsilon}$ and then we have $f \notin$ $V^{\gamma+2 \varepsilon}\left(\mathbb{R}^{n}\right)$.

Hence

$$
f \in V^{\gamma+}\left(\mathbb{R}^{n}\right)
$$

Lemma 6.2. Let $A$ be a compact subset of $\mathbb{R}^{n}$ and $f, g: A \rightarrow \mathbb{R}$ two continuous functions. Hence we have $\operatorname{osc}_{A}(f g) \leq \sup _{A}|f| \operatorname{osc}_{A}(g)+$ $\sup _{A}|g| o s c_{A}(f)$.

Proof of Theorem 4.2. For all $I$ as in Definition 3.1, the Lemma 6.2 gives $\operatorname{osc}_{I}(\eta f) \leq \operatorname{cosc}_{I}(f)+\sup |f| \operatorname{osc}_{I}(\eta)$. Let $0<\alpha \leq 1$. By summing over all $I$, multiplying by $2^{\nu(\alpha-n)}$ and taking the supremum over all $\nu \in \mathbb{N}_{0}$ we have $\|\eta f\|_{V^{\alpha}\left(\mathbb{R}^{n}\right)} \leq c\|f\|_{V^{\alpha}\left(\mathbb{R}^{n}\right)}+c^{\prime} \sup |f| \leq\left(c+c^{\prime}\right)\|f\|_{V^{\alpha}\left(\mathbb{R}^{n}\right)}$. Then the first equivalence of Theorem 4.1 gives the desired inequality.

Lemma 6.3 ([9], p. 19). For any $h>0$ and $k=\left(k_{1}, \ldots, k_{n}\right) \in \mathbb{Z}^{n}$, define $Q_{k}^{h}=\left\{x=\left(x_{1}, \ldots, x_{n}\right) \in \mathbb{R}^{n}\right.$ with $h k_{j} \leq x_{j}<h\left(k_{j}+1\right)$ for $j=1, \ldots, n\}$. Let $\Omega \subset \mathbb{R}^{n}$ be a compact set and $0<p \leq \infty$. Then, there exist three positive numbers $h_{0}, c_{1}$ and $c_{2}$ such that

$$
c_{1}\left(\sum_{k \in \mathbb{Z}^{n}}\left|\varphi\left(x_{k}\right)\right|^{p}\right)^{\frac{1}{p}} \leq h^{-\frac{n}{p}}\|\varphi\|_{L_{p}\left(\mathbb{R}^{n}\right)} \leq c_{2}\left(\sum_{k \in \mathbb{Z}^{n}}\left|\varphi\left(x_{k}\right)\right|^{p}\right)^{\frac{1}{p}}
$$

hold for all sets $\left(x_{k}\right)_{k \in \mathbb{Z}^{n}}$ with $x_{k} \in Q_{k}^{h}$, all $h$ with $0<h<h_{0}$, and all $\varphi \in S^{\Omega}\left(\mathbb{R}^{n}\right)$. (Modification if $p=\infty$ ).

Lemma 6.4 ([9], p. 22). Let $\Omega \subset \mathbb{R}^{n}$ be a compact set. Let $0<p \leq q \leq$ $\infty$ and $\alpha \in\left(\mathbb{N}_{0}\right)^{n}$ a multi-index. Then there exists a positive constant $c$ such that for all $f \in L_{p}^{\Omega}\left(\mathbb{R}^{n}\right)$ we have

$$
\left\|D^{\alpha} f\right\|_{L_{p}\left(\mathbb{R}^{n}\right)} \leq c\|f\|_{L_{p}\left(\mathbb{R}^{n}\right)} .
$$

Lemma 6.5 ([9], p. 129). Let $0<p_{0} \leq p_{1} \leq \infty, 0<q \leq \infty$ and $-\infty<s_{1} \leq s_{0}<\infty$. If $s_{0}-\frac{n}{p_{0}}=s_{1}-\frac{n}{p_{1}}$ then we have the embedding

$$
B_{p_{0}, q}^{s_{0}}\left(\mathbb{R}^{n}\right) \hookrightarrow B_{p_{1}, q}^{s_{1}}\left(\mathbb{R}^{n}\right) .
$$


Lemma 6.6 ([9], p. 195). Let $\varphi \in C_{0}^{\infty}\left(\mathbb{R}^{n}\right)$. Let $0<p_{0} \leq p_{1} \leq \infty$, $0<q \leq \infty$ and $s \in \mathbb{R}$. Then $f \mapsto \varphi f$ yields a continuous linear mapping from $B_{p_{1}, q}^{s}\left(\mathbb{R}^{n}\right)$ into $B_{p_{0}, q}^{s}\left(\mathbb{R}^{n}\right)$.

Definition 6.2. If $G \subset \mathbb{R}^{n}$ is a bounded set we define $S^{G}\left(\mathbb{R}^{n}\right)=$ $\left\{\psi \in S\left(\mathbb{R}^{n}\right)\right.$ with $\left.\operatorname{supp}(F \psi) \subset G\right\}$ and $L_{p}^{G}\left(\mathbb{R}^{n}\right)=\left\{f \in L_{p}\left(\mathbb{R}^{n}\right)\right.$ with $\operatorname{supp}(F f) \subset G\}$ for $0<p \leq \infty$.

Lemma 6.7. Let $0<p<\infty$ and consider $G, A \subset \mathbb{R}^{n}$ be non-empty bounded sets, with $G$ open. Then $S^{G}\left(\mathbb{R}^{n}\right)$ is dense in $L_{p}^{G}\left(\mathbb{R}^{n}\right)$ with the norm $\|\cdot\|_{L_{p}\left(\mathbb{R}^{n}\right)}+\sup _{A}|\cdot|$.

Proof of Lemma 6.7. Consider $f \in L_{p}^{G}\left(\mathbb{R}^{n}\right)$. Let $\zeta \in S\left(\mathbb{R}^{n}\right)$ with $\zeta(0)=1,|\zeta(x)| \leq 1, \forall x \in \mathbb{R}^{n}$ and with $\operatorname{supp}(F \zeta)$ compact. It is easy to find such a $\zeta$, because a translation of $\zeta$ and a multiplication by a scalar $k \in \mathbb{C} \backslash\{0\}$ preserves $\operatorname{supp}(F \zeta)$.

For all $k \in \mathbb{N}$ let $\psi_{k}(x)=f(x) \zeta(x / k), \forall x \in \mathbb{R}^{n}$. Then by the PaleyWiener-Schwartz theorems ([9], p. 13) we have $\psi_{k} \in S\left(\mathbb{R}^{n}\right), \forall k \in \mathbb{N}$. Because $G$ is open, for $k$ sufficiently large we have $\psi_{k} \in S^{G}\left(\mathbb{R}^{n}\right)$. Let $\varepsilon>0$ and $M_{\varepsilon}>0$ with $\int_{|t| \geq M_{\varepsilon}}|f(t)|^{p} d t \leq \frac{1}{2}\left(\frac{\varepsilon}{2}\right)^{p}$.

Let $k_{\varepsilon} \in \mathbb{N}$ with $|\zeta(x / k)-1|^{p} \leq \frac{\varepsilon^{p} / 2}{1+\int_{|t| \leq M_{\varepsilon}}|f(t)|^{p} d t}$ for all $x \in \mathbb{R}^{n}$ with $|x| \leq M_{\varepsilon}$ or $x \in A$, and for all $k \geq k_{\varepsilon}$. We observe that by the PaleyWiener-Schwartz theorem ([9], p. 13) we have $f$ bounded on $A$. Hence $\sup _{A}\left|\psi_{k}-f\right| \leq \varepsilon \sup _{A}|f|, \forall k \geq k_{\varepsilon}$ and $\left\|\psi_{k}-f\right\|_{L_{p}\left(\mathbb{R}^{n}\right)} \leq \varepsilon$.

Lemma 6.8. Let $I$ be a $n$-cube as in Definition 3.1 and $\varphi_{j}$ as in Definition 2.5 for all $j \in \mathbb{N}_{0}$. Let $f: \mathbb{R}^{n} \rightarrow \mathbb{R}$ be a continuous function with $f \in S^{\prime}\left(\mathbb{R}^{n}\right)$. Then we have

$$
(2 \pi)^{\frac{n}{2}} \operatorname{osc}_{I}(f) \leq \sum_{j \geq 0} \operatorname{osc}_{I} \operatorname{Re}\left(\varphi_{j} * f\right) .
$$

Proof of Lemma 6.8. We make only a layout of the proof. For all $k \in \mathbb{N}$ let $f_{k}=\sum_{j=0}^{k} \varphi_{j} * f, h_{k}=\sum_{j=0}^{k} \sup _{I} \operatorname{Re}\left(\varphi_{j} * f\right)$ and $h_{k}^{\prime}=\sum_{j=0}^{k} \inf _{I} \operatorname{Re}\left(\varphi_{j} * f\right)$.

Let $\varepsilon>0$. Then, by the convergence $\lim _{k \rightarrow \infty} f_{k}=(2 \pi)^{\frac{n}{2}} f$ with the weak topology in $S^{\prime}\left(\mathbb{R}^{n}\right)$, we deduce that $\exists k_{\varepsilon} \in \mathbb{N}$ such that we have $(2 \pi)^{\frac{n}{2}} \sup _{I} f \leq h_{k}+\varepsilon, \forall k \geq k_{\varepsilon}$. By this formula and the counterpart $(2 \pi)^{\frac{n}{2}} \inf _{I} f \geq h_{k}^{\prime}-\varepsilon, \forall k \geq k_{\varepsilon}^{\prime}$ for some $k_{\varepsilon}^{\prime} \in \mathbb{N}$, we have the desired inequality. 
Proof of Theorem 5.1. By [9], p. 131 we have $f$ continuous. Let $0<p \leq 1$. Then $\gamma \geq 1$ and by the Lemma 6.5 we have also $\|f\|_{B_{1,1}^{\gamma+n-\frac{n}{p}}\left(\mathbb{R}^{n}\right)} \leq c\|f\|_{B_{p, 1}^{\gamma}\left(\mathbb{R}^{n}\right)}$. Because $\gamma+n-\frac{n}{p} \geq \frac{n}{1}$ we can assume $1 \leq p<\infty$.

(i) Let $j \in \mathbb{N}_{0}$ and $\nu \in \mathbb{N}_{0}$. Let $G_{j}=\left\{\xi \in \mathbb{R}^{n}:|\xi|<2^{j+2}\right\}$ and $\psi_{j} \in S^{G_{j}}\left(\mathbb{R}^{n}\right)$. Let $|I|=2^{-\nu n}, I$ as in Definition 3.1.

Let $\sum^{\prime}$ and $\sum^{\prime j}$ be the sums over the cubes $I$ restricted to the cube $[-m, m]^{n}$ and $\left[-m 2^{j}, m 2^{j}\right]^{n}$, respectively.

(i1) Let $\nu \leq j$. We have

$$
\operatorname{osc}_{I}\left(\operatorname{Re} \psi_{j}\right) \leq 2 \sup _{I}\left|\psi_{j}\right| .
$$

Let $j_{0} \in \mathbb{N}_{0}$. By Hölder's inequality and because $\nu \leq j$ we have

$$
\begin{aligned}
\sum_{|I|=2^{-\nu n}}^{\prime} \sup _{I}\left|\psi_{j}\right| & \leq\left(2^{\nu} 2 m\right)^{n \frac{p-1}{p}}\left(\sum_{|I|=2^{-\nu n}}^{\prime} \sup _{I}\left|\psi_{j}\right|^{p}\right)^{\frac{1}{p}} \\
& \leq c_{m} 2^{\nu n \frac{p-1}{p}}\left(\sum_{|I|=2^{-j_{0} n}}^{\prime j} \sup _{I}\left|\psi_{j}\left(2^{-j} \cdot\right)\right|^{p}\right)^{\frac{1}{p}} .
\end{aligned}
$$

Taking $j_{0}$ sufficiently large then by the Lemma 6.3 the factor $(.)^{\frac{1}{p}}$ can be estimated from above by

$$
c 2^{\frac{j_{0} n}{p}}\left\|\psi_{j}\left(2^{-j} \cdot\right)\right\|_{L_{p}\left(\mathbb{R}^{n}\right)}=c^{\prime} 2^{j \frac{n}{p}}\left\|\psi_{j}\right\|_{L_{p}\left(\mathbb{R}^{n}\right)} .
$$

Because $\nu \leq j$ and $\gamma \geq \frac{n}{p}$ we have

$$
\nu \gamma+(j-\nu) \frac{n}{p} \leq j \gamma
$$

Then we have

$$
2^{\nu(\gamma-n)} \sum_{|I|=2^{-\nu n}}^{\prime} \operatorname{osc}_{I}\left(\operatorname{Re} \psi_{j}\right) \leq c_{m} 2^{j \gamma}\left\|\psi_{j}\right\|_{L_{p}\left(\mathbb{R}^{n}\right)} .
$$

(i2) Let $\nu \geq j$. We have by the mean value Theorem

$$
\operatorname{osc}_{I}\left(\operatorname{Re}_{j}\right) \leq 2^{-\nu} \sum_{i=1}^{n} \sup _{I}\left|\frac{\partial}{\partial x_{i}} \operatorname{Re} \psi_{j}\right| .
$$


Let $i \in\{1, \ldots, n\}$. By Hölder's inequality we have $\sum_{|I|=2^{-\nu n}}^{\prime} \sup _{I}\left|\frac{\partial}{\partial x_{i}} \operatorname{Re} \psi_{j}\right| \leq\left(2^{\nu} 2 m\right)^{n \frac{p-1}{p}}\left(\sum_{|I|=2^{-\nu n}}^{\prime} \sup _{I}\left|\frac{\partial}{\partial x_{i}} \psi_{j}\right|^{p}\right)^{\frac{1}{p}}$.

Let $\nu_{0} \in \mathbb{N}_{0}$. Because $\nu \geq j$ the last sum can be estimated from above by

$$
\sum_{|I|=2^{-\left(\nu+\nu_{0}-j\right) n}}^{\prime j} \sup _{I}\left|\left(\frac{\partial}{\partial x_{i}} \psi_{j}\right)\left(2^{-j} \cdot\right)\right|^{p} .
$$

Taking $\nu_{0}$ sufficiently large then by the Lemma 6.3 the factor (.) ${ }^{\frac{1}{p}}$ can be estimated from above by

$$
c 2^{\left(\nu+\nu_{0}-j\right) \frac{n}{p}}\left\|\left(\frac{\partial}{\partial x_{i}} \psi_{j}\right)\left(2^{-j} \cdot\right)\right\|_{L_{p}\left(\mathbb{R}^{n}\right)} .
$$

By the Lemma 6.4 this last norm can be estimated from above by

$$
c\left\|2^{j} \psi_{j}\left(2^{-j} \cdot\right)\right\|_{L_{p}\left(\mathbb{R}^{n}\right)}=c 2^{j} 2^{j \frac{n}{p}}\left\|\psi_{j}\right\|_{L_{p}\left(\mathbb{R}^{n}\right)} .
$$

Because $\nu \geq j$ we have

$$
\nu \min (1, \gamma)-\nu+j \leq j \min (1, \gamma) .
$$

Then we obtain

$$
2^{\nu(\min (1, \gamma)-n)} \sum_{|I|=2^{-\nu n}}^{\prime} \operatorname{osc}_{I}\left(\operatorname{Re} \psi_{j}\right) \leq c_{m} 2^{j \min (1, \gamma)}\left\|\psi_{j}\right\|_{L_{p}\left(\mathbb{R}^{n}\right)} .
$$

(ii) Let $\nu \in \mathbb{N}_{0}, j \in \mathbb{N}_{0}$ and $\varphi_{j}$ as in Definition 2.5.

We have $\varphi_{j} * f \in L_{p}^{G_{j}}\left(\mathbb{R}^{n}\right)$. Therefore, by (i) and Lemma 6.7 we have

$$
2^{\nu(\min (1, \gamma)-n)} \sum_{|I|=2^{-\nu n}}^{\prime} \operatorname{osc}_{I} \operatorname{Re}\left(\varphi_{j} * f\right) \leq c_{m} 2^{j \gamma}\left\|\varphi_{j} * f\right\|_{L_{p}\left(\mathbb{R}^{n}\right)} .
$$

(iii) Let $\nu \in \mathbb{N}_{0}$. By Lemma 6.8 we have

$$
2^{\nu(\min (1, \gamma)-n)} \sum_{|I|=2^{-\nu n}}^{\prime}{ }_{\sin } f \leq c_{m}\|f\|_{B_{p, 1}^{\gamma}\left(\mathbb{R}^{n}\right)} .
$$


Of course, we may replace in the obtained inequality $\sum^{\prime}$ by $\sum$. Then by taking the $\sup _{\nu \geq 0}$ on the left hand side we have the desired inequality.

Proof of Corollary 5.2. We have $f \in \bigcap_{\frac{n}{p} \leq \sigma<\gamma} B_{p, 1}^{\sigma}\left(\mathbb{R}^{n}\right)$ and by Theorem 5.1 we have $f \in V^{\min (1, \gamma)-}\left(\mathbb{R}^{n}\right)$ in case $0<p<\infty$. On the other hand, if $p=\infty$ then the double embedding $B_{\infty}^{\gamma-}\left(\mathbb{R}^{n}\right)=C^{\gamma-}\left(\mathbb{R}^{n}\right)$ and the remark (b) of Definition 4.2 gives $f \in V^{\gamma-}\left(\mathbb{R}^{n}\right)$. Hence Theorem 4.1 completes the proof.

Definition 6.3. Let $\rho>0$ and $x \in \mathbb{R}^{n}$. Then we define $B_{\rho} x=\{t \in$ $\left.\mathbb{R}^{n}:|t-x| \leq \rho\right\}$, the closed ball centered in $x$ and with radius $\rho$.

Definition 6.4. Let $f: \mathbb{R}^{n} \rightarrow \mathbb{R}$ be a measurable and bounded function with a compact support. Let $0<\varepsilon<1, j \in \mathbb{N}$ and $\varphi_{j}$ as in Definition 2.5.

(i) We define

$$
\left(\varphi_{j} * * f\right)(x)=\int_{|t| \leq 2^{-j+[j \varepsilon]}} \varphi_{j}(t) f(x-t) d t, \forall x \in \mathbb{R}^{n} .
$$

(ii) (ii1) We define $\left(\prod_{i=1}^{n}\left[a_{i}, a_{i}+2^{-j}\right]\right)^{\#}=\prod_{i=1}^{n}\left[a_{i}-2^{-j+[j \varepsilon]}, a_{i}+2^{-j}+\right.$ $\left.2^{-j+[j \varepsilon]}\right]$, where $a_{i} \in \mathbb{R}, \forall i=1, \ldots, n$.

(ii2) Let $|I|=2^{-j n}, I$ be as in Definition 3.1. We define $f_{I}(x)=$ $f(x)-\frac{1}{\mid I^{\# \mid}} \int_{I^{\#}} f(t) d t, \forall x \in \mathbb{R}^{n}$.

(ii3) Let $|I|=2^{-j n}, I$ be as in Definition 3.1, and $I^{o}$ be the interior of $I$. We define almost everywhere

$$
\left(\varphi_{j} * * * f\right)(x)=\int_{|t| \leq 2^{-j+[j \varepsilon]}} \varphi_{j}(t) f_{I}(x-t) d t, \forall x \in I^{o} .
$$

Remark. (a) We have $\int_{I^{\#}} f_{I}(t) d t=0$.

(b) For $j \in \mathbb{N}$ we have $\varphi_{j} * * f$ continuous and $\varphi_{j} * * * f$ continuous almost everywhere.

Lemma 6.9. Let $f: \mathbb{R}^{n} \rightarrow \mathbb{R}$ be a measurable and bounded function with a compact support. Let $0<p \leq \infty, 0<q \leq \infty$ and $s \in \mathbb{R}$.

Let $j_{0} \in \mathbb{N}$ and $0<\varepsilon<1$. Then we have the equivalences

$$
\begin{aligned}
f \in B_{p, q}^{s}\left(\mathbb{R}^{n}\right) & \Longleftrightarrow \sum_{j \geq j_{0}}\left(2^{j s}\left\|\varphi_{j} * * f\right\|_{L_{p}\left(\mathbb{R}^{n}\right)}\right)^{q}<\infty \\
& \Longleftrightarrow \sum_{j \geq j_{0}}\left(2^{j s}\left\|\varphi_{j} * * * f\right\|_{L_{p}\left(\mathbb{R}^{n}\right)}\right)^{q}<\infty .
\end{aligned}
$$

(Standard modification if $q=\infty$.) 
Proof of Lemma 6.9. Let $R, L>0$ such that supp $f \subset B_{R}(0, \ldots, 0)$ and $\sup |f| \leq L$. Let $\varphi$ and $\varphi_{j}$ as in Definition 2.5 for all $j \in \mathbb{N}_{0}$. By the PaleyWiener-Schwartz theorems ([9], p. 13) we have $\varphi_{j} * f \in S\left(\mathbb{R}^{n}\right), \forall j \in \mathbb{N}_{0}$ and then $\left\|\varphi_{j} * f\right\|_{L_{p}\left(\mathbb{R}^{n}\right)}<\infty, \forall j \in \mathbb{N}_{0}$.

Consider $k>0$. Then we have $|x|^{k}|\varphi(x)| \leq c_{k}, \forall x \in \mathbb{R}^{n}$. Let $j \in \mathbb{N}$ sufficiently large.

(i) Let $x \in \mathbb{R}^{n}$. We have $\left(\varphi_{j} * * f\right)(x)=0$ for $|x| \geq R+\frac{1}{2}$ and $\left(\varphi_{j} * * * f\right)(x)=0$ for $|x| \geq R+2$.

(ii) For all $x \in \mathbb{R}^{n}$ with $|x| \geq R+1$ we have $\left|\left(\varphi_{j} * f\right)(x)\right| \leq$ $c_{k, R, L} 2^{j(n-k)}|x|^{-k}$.

(iii) Let $k \geq n+1$ and $x \in \mathbb{R}^{n}$. Then we have $\left|\left(\varphi_{j} * * f\right)(x)-\left(\varphi_{j} * f\right)(x)\right| \leq$ $c_{k, L} 2^{j \frac{\varepsilon}{2}(n-k)}$.

(iv) Let $k \geq n+1$ and $x \in \mathbb{R}^{n}$. Then we have

$$
\begin{aligned}
\left|\left(\varphi_{j} * * * f\right)(x)-\left(\varphi_{j} * * f\right)(x)\right| & \leq\left|\int_{|t| \leq 2^{-j+[j \varepsilon]}} L \varphi_{j}(t) d t\right| \\
& =L\left|\int_{|t| \geq 2^{-j+[j \varepsilon]}} \varphi_{j}(t) d t\right| \\
& \leq L \int_{|t| \geq 2^{-j+[j \varepsilon]}}\left|\varphi_{j}(t)\right| d t \\
& \leq L \int_{|t| \geq 2^{-j+j \frac{\varepsilon}{2}}}\left|\varphi_{j}(t)\right| d t \\
& \leq L \int_{|t| \geq 2^{j \frac{\varepsilon}{2}}}|\varphi(t)| d t \\
& \leq c_{k} L \int_{|t| \geq 2^{j \frac{\varepsilon}{2}}}|t|^{-k} d t \\
& =c_{k, L} 2^{j \frac{\varepsilon}{2}(n-k)} \int_{|t| \geq 1}|t|^{-k} d t \\
& \leq c_{k, L}^{\prime} 2^{j \frac{\varepsilon}{2}(n-k)} .
\end{aligned}
$$

(v) Then by (i)-(iv), if we take $k \geq n+1$ sufficiently large we have the desired equivalences.

Proof of Theorem 5.3. Of course $f$ is bounded and measurable, and then $f \in S^{\prime}\left(\mathbb{R}^{n}\right)$. Let $R, L>0$ such that supp $f \subset B_{R}(0, \ldots, 0)$ and $\sup |f| \leq L$. Let $0<\varepsilon<1$. We make use of the Lemma 6.9.

(i) Let $0<p \leq 1$ and $j \in \mathbb{N}$. 
By Hölder's inequality we have

$$
\begin{aligned}
\int_{|x| \leq R+1}\left|\left(\varphi_{j} * * f\right)(x)\right|^{p} d x \leq & \left(\int_{|x| \leq R+1}\left(\left|\left(\varphi_{j} * * f\right)(x)\right|^{p}\right)^{\frac{1}{p}}\right)^{p} \\
& \times\left(\int_{|x| \leq R+1} 1^{\frac{1}{1-p}}\right)^{1-p} .
\end{aligned}
$$

Then we obtain

$$
\left\|\varphi_{j} * * f\right\|_{L_{p}\left(\mathbb{R}^{n}\right)} \leq c_{R}\left\|\varphi_{j} * * f\right\|_{L_{1}\left(\mathbb{R}^{n}\right)} .
$$

Hence we may assume $1 \leq p<\infty$.

(ii) Let $1 \leq p<\infty$ and $j \in \mathbb{N}$ sufficiently large. Let $|I|=2^{-j n}, I$ as in Definition 3.1.

Then for $x \in I$ we have

$$
\begin{aligned}
\left|\left(\varphi_{j} * * * f\right)(x)\right| & \leq \int_{|t| \leq 2^{-j+[j \varepsilon]}}\left|\varphi_{j}(t) f_{I}(x-t)\right| d t \leq c 2^{j n} 2^{(-j+[j \varepsilon]) n} \sup _{I^{\#}}\left|f_{I}\right| \\
& \leq c 2^{j \varepsilon n} \sup _{I \#}\left|f_{I}\right| .
\end{aligned}
$$

Therefore we obtain

$$
\sup _{I}\left|\varphi_{j} * * * f\right| \leq c 2^{j \varepsilon n} \sup _{I \#}\left|f_{I}\right| .
$$

By the remark (a) of Definition 6.4 we have $\sup _{I^{\#}} f_{I} \inf _{I^{\#}} f_{I} \leq 0$, and therefore $\sup _{I \#}\left|f_{I}\right| \leq \sup _{I \#} f_{I}-\inf _{I \#} f_{I}$. The right hand side of last inequality can be estimated from above by $\sum_{I^{\prime}} o s c_{I^{\prime}}\left(f_{I}\right)=\sum_{I^{\prime}} o s c_{I^{\prime}}(f)$, where the last two sums are taken over all $\left(1+2^{[j \varepsilon]} 2\right)^{n} n$-cubes $I^{\prime} \subset I^{\#},\left|I^{\prime}\right|=2^{-j n}, I^{\prime}$ as in Definition 3.1.

Hence

$$
\begin{aligned}
\left\|\varphi_{j} * * * f\right\|_{L_{p}\left(\mathbb{R}^{n}\right)} & \leq\left(\sum_{|I|=2^{-j n}}|I| \sup _{I}\left|\varphi_{j} * * * f\right|^{p}\right)^{\frac{1}{p}} \\
& \leq c 2^{-j \frac{n}{p}}\left(\sum_{|I|=2^{-j n}}\left(2^{j \varepsilon n} \sum_{I^{\prime}} \operatorname{osc}_{I^{\prime}}(f)\right)^{p}\right)^{\frac{1}{p}}
\end{aligned}
$$




$$
\leq c 2^{j \varepsilon n} 2^{-j \frac{n}{p}}\left(\sum_{|I|=2^{-j n}}\left(1+2^{[j \varepsilon]} 2\right)^{n p} \sum_{I^{\prime}}\left(\operatorname{osc}_{I^{\prime}}(f)\right)^{p}\right)^{\frac{1}{p}} .
$$

This value can be estimated from above by

$$
\begin{aligned}
& c 2^{j 2 \varepsilon n} 2^{-j \frac{n}{p}}\left(\sum_{|I|=2^{-j n}} \sum_{I^{\prime}}\left(\operatorname{osc}_{I^{\prime}}(f)\right)^{p}\right)^{\frac{1}{p}} \\
& \leq c_{L} 2^{j 2 \varepsilon n} 2^{-j \frac{n}{p}}\left(\sum_{|I|=2^{-j n}} \sum_{I^{\prime}} o s c_{I^{\prime}}(f)\right)^{\frac{1}{p}} \\
& \leq c_{L} 2^{j 2 \varepsilon n} 2^{-j \frac{n}{p}}\left(\left(1+2^{[j \varepsilon]} 2\right)^{n} O s c(j, f)\right)^{\frac{1}{p}} \\
& \leq c_{L}^{\prime} 2^{j 2 \varepsilon n} 2^{j \frac{\varepsilon n}{p}} 2^{-j \frac{n}{p}} O s c(j, f)^{\frac{1}{p}} .
\end{aligned}
$$

Because $f \in V^{\gamma^{\prime} p}$ this value can be estimated from above by

$$
c_{L} 2^{j 3 \varepsilon n} 2^{-j \frac{n}{p}}\left(c 2^{j\left(n-\gamma^{\prime} p\right)}\right)^{\frac{1}{p}}=c_{L}^{\prime} 2^{j 3 \varepsilon n} 2^{-j \gamma^{\prime}} .
$$

Then by taking $\varepsilon>0$ sufficiently small we get

$$
2^{j \gamma}\left\|\varphi_{j} * * * f\right\|_{L_{p}\left(\mathbb{R}^{n}\right]} \leq c_{L} .
$$

Lemma 6.10. Let $r>1$. Then by the mean value Theorem, for some $c \in(r, r+1)$ we have

$$
\left|\frac{1}{\ln (r+1)}-\frac{1}{\ln r}\right|=\frac{1}{c \ln ^{2} c} \geq \frac{1}{(r+1) \ln ^{2}(r+1)} .
$$

Hence for $m \in \mathbb{N}$ with $m \geq 2$ we obtain

$$
\begin{aligned}
\sum_{j=2}^{m} \frac{1}{2^{j}\left|\frac{1}{\ln (m+1)}-\frac{1}{\ln j}\right|} & \leq \sum_{j \geq 2} \frac{1}{2^{j}\left|\frac{1}{\ln (j+1)}-\frac{1}{\ln j}\right|} \\
& \leq \sum_{j \geq 2} \frac{(j+1) \ln ^{2}(j+1)}{2^{j}}<\infty .
\end{aligned}
$$

Proof of Theorem 5.6. We will restrict us to the case $n=1$. For $n \in \mathbb{N} \backslash\{1\}$ the proof is analogous, although with more fastidious calculus. In corresponding parts (i) and (ii) we use essentially the fact that the case 
$n=1$ remains valid if we replace $\Theta$ by $\Theta\left(., x_{2}, \ldots, x_{n}\right)$, for all (some in ii-case) fixed points $\left(x_{2}, \ldots, x_{n}\right) \in \mathbb{R}^{n-1}$.

(i) We prove that $\Theta$ is bounded on $\mathbb{R}$ and continuous on $\mathbb{R} \backslash\{0\}$ :

We have $|x \psi(x)| \leq c, \forall x \in \mathbb{R}$.

(i1) Let $x \leq 0$ and $j_{1} \in \mathbb{N} \backslash\{1,2\}$.

Then

$$
\begin{aligned}
\sum_{j \geq j_{1}}\left|\Theta_{j}(x)\right| & =\sum_{j \geq j_{1}}\left|\psi\left(2^{j+j_{0}}\left(x-\frac{1}{\ln j}\right)\right)\right| \\
& \leq \sum_{j \geq j_{1}} \frac{c}{2^{j+j_{0}}\left|x-\frac{1}{\ln j}\right|} \\
& \leq \sum_{j \geq j_{1}} c^{\prime} 2^{-j} \ln j .
\end{aligned}
$$

Hence $\sum_{j \geq j_{1}} \Theta_{j}$ is bounded on $(-\infty, 0]$ and converges uniformly on $(-\infty, 0]$.

In particular $\Theta$ is continuous on $(-\infty, 0)$.

(i2) Let $j_{1} \in \mathbb{N} \backslash\{1,2\}$.

Let $x \geq \frac{1}{\ln j_{1}}$.

Then

$$
\begin{aligned}
\sum_{j \geq j_{1}+1}\left|\Theta_{j}(x)\right| & =\sum_{j \geq j_{1}+1}\left|\psi\left(2^{j+j_{0}}\left(x-\frac{1}{\ln j}\right)\right)\right| \\
& \leq \sum_{j \geq j_{1}+1} \frac{c}{2^{j+j_{0}}\left|x-\frac{1}{\ln j}\right|} \\
& \leq c_{j_{1}} \sum_{j \geq j_{1}+1} 2^{-j} .
\end{aligned}
$$

Hence $\sum_{j \geq 3} \Theta_{j}$ converges uniformly on $\left[\frac{1}{\ln j_{1}}, \infty\right)$.

In particular $\Theta$ is continuous on $(0, \infty)$.

(i3) Let $x>0$.

Let $j(x)=\min \left\{j \in \mathbb{N} \backslash\{1,2\}\right.$ with $\left.x \geq \frac{1}{\ln j}\right\}$.

We have

$$
\begin{aligned}
\sum_{j \geq 3}\left|\Theta_{j}(x)\right| & \leq 2 \sup |\psi|+\sum_{j=3}^{j(x)-2}\left|\Theta_{j}(x)\right|+\sum_{j \geq j(x)+1}\left|\Theta_{j}(x)\right| \\
& \leq 2 \sup |\psi|+\sum_{j=3}^{j(x)-2} \frac{c}{2^{j+j_{0}}\left|\frac{1}{\ln (j(x)-1)}-\frac{1}{\ln j}\right|}
\end{aligned}
$$




$$
+\sum_{j \geq j(x)+1} \frac{c}{2^{j+j_{0}}\left|\frac{1}{\ln j(x)}-\frac{1}{\ln j}\right|} .
$$

The second sum on the right hand side can be estimated from above by $\frac{2^{-j(x)}}{\ln j(x)}-\frac{1}{\ln (j(x)+1)} \sum_{j \geq 1} \frac{c}{2^{j+j_{0}}}$, and the factor before the sum tends to 0 when $x \rightarrow 0^{+}$.

On the other hand by the Lemma 6.10 we have

$$
\sum_{j=3}^{j(x)-2} \frac{1}{2^{j}\left|\frac{1}{\ln (j(x)-1)}-\frac{1}{\ln j}\right|} \leq c<\infty .
$$

Hence we have

$$
\sum_{j \geq 3}\left|\Theta_{j}(x)\right| \leq 2 \sup |\psi|+c 2^{-j_{0}}, \forall x>0 .
$$

Therefore $\Theta$ is bounded on $(0, \infty)$.

(ii) We may assume $\psi(0)=18$. For the general case, in what follows we need only to change some constants by the factor $\psi(0) / 18 \neq 0$.

Let $j_{1} \in \mathbb{N} \backslash\{1,2\}$ and $j_{0}$ sufficiently large.

We have

$$
\begin{aligned}
\Theta\left(\frac{1}{\ln j_{1}}\right) & =\Theta_{j_{1}}\left(\frac{1}{\ln j_{1}}\right)+\sum_{j \geq 3 \wedge j \neq j_{1}} \Theta_{j}\left(\frac{1}{\ln j_{1}}\right) \\
& \geq \psi(0)-\sum_{j \geq 3 \wedge j \neq j_{1}}\left|\Theta_{j}\left(\frac{1}{\ln j_{1}}\right)\right| .
\end{aligned}
$$

By calculations like (i3) the last sum can be evaluated from above by $c 2^{-j_{0}} \leq 1$. Then $\Theta\left(\frac{1}{\ln j_{1}}\right) \geq 17$.

Also by calculations like in (i3) we have

$$
\Theta\left(\frac{\frac{1}{\ln j_{1}}+\frac{1}{\ln \left(j_{1}+1\right)}}{2}\right) \leq c 2^{-j_{0}} \leq 1 .
$$

Therefore

$$
\sup _{x, y \in\left[\frac{1}{\ln \left(j_{1}+1\right)}, \frac{1}{\ln j_{1}}\right]}\left|\Theta_{0}(x)-\Theta_{0}(y)\right| \geq \frac{17}{\ln j_{1}}-\frac{1}{\ln j_{1}}=\frac{2^{4}}{\ln j_{1}} .
$$

Let $0<\varepsilon<1$. 
For every $\nu \in \mathbb{N}_{0}$ let $k(\nu)=\left[2^{\nu(1-\varepsilon)}\right]$. Then $k(\nu) \geq \frac{1}{2} 2^{\nu(1-\varepsilon)}, \forall \nu \in$ $\mathbb{N}_{0}$.

Let $\nu_{0} \in \mathbb{N}_{0}$ such that $2 e^{-2^{\nu \varepsilon}} 2^{\nu(1-2 \varepsilon)} \leq 1, \forall \nu \geq \nu_{0}$.

Then $\left|\left(\frac{1}{\ln t}\right)^{\prime}\right| 1 \leq \frac{1}{2} 2^{-\nu}$ if $\frac{1}{\ln t} \leq k(\nu) 2^{-\nu}$ with $t \geq 3$ and $\nu \geq \nu_{0}$.

Here $(.)^{\prime}$ represents the derivative operator.

Let $\nu_{1} \geq \nu_{0}$ with $k\left(\nu_{1}\right) 2^{-\nu_{1}} \leq \frac{1}{\ln 3}$. If $\nu \geq \nu_{1}$ then for every $k \in\{1, \ldots, k(\nu)\}$ there exists $j_{\nu, k} \in \mathbb{N} \backslash\{1,2\}$ such that

$$
\left\{\frac{1}{\ln j_{\nu, k}}, \frac{1}{\ln \left(j_{\nu, k}+1\right)}\right\} \subset\left[(k-1) 2^{-\nu}, k 2^{-\nu}\right] .
$$

Hence

$$
\begin{aligned}
\operatorname{osc}_{\left[(k-1) 2^{-\nu}, k 2^{-\nu}\right]}\left(\Theta_{0}\right) & \geq \frac{2^{4}}{\ln j_{\nu, k}} \\
& \geq 2^{4}(k-1) 2^{-\nu}, \forall k \in\{1, \ldots, k(\nu)\}, \forall \nu \geq \nu_{1} .
\end{aligned}
$$

Let $I$ as in Definition 3.1. We have

$$
\operatorname{Osc}\left(\nu, \Theta_{0}\right) \geq \sum_{k=1}^{k(\nu)} 2^{4}(k-1) 2^{-\nu}, \forall \nu \geq \nu_{1} .
$$

Then $\operatorname{Osc}\left(\nu, \Theta_{0}\right) \geq 2^{4} \frac{(k(\nu)-1) k(\nu)}{2} 2^{-\nu}, \forall \nu \geq \nu_{1}$.

For $\nu \geq \nu_{2}$ with $\nu_{2} \geq \nu_{1}$ sufficiently large we have

$$
\operatorname{Osc}\left(\nu, \Theta_{0}\right) \geq 2^{2}(k(\nu))^{2} 2^{-\nu} \geq 2^{\nu(1-2 \varepsilon)} .
$$

Hence if we take $0<2 \varepsilon<1$ and use the lower counterpart of the second equality of the Lemma 6.1 (see Remark 4.3) then we have $\underline{\operatorname{dim}}_{B} \Gamma\left(\Theta_{0}\right) \geq 2-2 \varepsilon$. Hence we obtain $\operatorname{dim}_{B} \Gamma\left(\Theta_{0}\right)=2$.

(iii) Here we choose $a>0$. Let $\varphi$ be as in Definition 2.5, and with $\operatorname{supp}(F \varphi) \subset\left\{\xi \in \mathbb{R}^{n}: \frac{1}{2}(1+a) \leq|\xi| \leq 2(1-a)\right\}$ and $(F \varphi)(\xi)=1$ if $1-a \leq|\xi| \leq 1+a$, for some $a>0$.

Let $s<\frac{1}{p}$ and $0<q \leq \infty$. In the next first equality we make use of the continuity (see [7], p. 18) of the convolution operator in $S^{\prime}(\mathbb{R})$.

We have

$$
\begin{aligned}
\sum_{j \geq 0}\left(2^{j s}\left\|\varphi_{j} * \Theta\right\|_{L_{p}(\mathbb{R})}\right)^{q} & =(2 \pi)^{\frac{n}{2}} \sum_{j \geq 3}\left(2^{\left(j+j_{0}\right) s}\left\|\Theta_{j}\right\|_{L_{p}(\mathbb{R})}\right)^{q} \\
& =(2 \pi)^{\frac{n}{2}} \sum_{j \geq 3}\left(2^{\left(j+j_{0}\right) s} 2^{-\left(j+j_{0}\right) \frac{1}{p}}\|\psi\|_{L_{p}(\mathbb{R})}\right)^{q}
\end{aligned}
$$




$$
=c \sum_{j \geq 3} 2^{\left(j+j_{0}\right)\left(s-\frac{1}{p}\right) q}<\infty .
$$

(Standard modification if $p, q=\infty$.)

Hence we have $\Theta \in B_{p, q}^{s}(\mathbb{R})$. By the Lemma 6.6 we have also $\Theta_{0} \in B_{p, q}^{s}(\mathbb{R})$.

Proof of Theorem 5.8. The proof is similar to the one of the Theorem 5.6 , although more complicated. We make a layout for the part (c). We need modification if $h=n-1$ in the case $n \in \mathbb{N} \backslash\{1\}$.

We choose $a>0$. Let $\varphi$ be as in Definition 2.5, and with $\operatorname{supp}(F \varphi) \subset$ $\left\{\xi \in \mathbb{R}^{n}: \frac{1}{2}(1+2 a) \leq|\xi| \leq 2(1-2 a)\right\}$ and $(F \varphi)(\xi)=1$ if $1-2 a \leq|\xi| \leq$ $1+2 a$, for some $a>0$.

(i) For $n=1,0<q \leq \infty$ and $s \in \mathbb{R}$ we have the equivalence

$$
\Theta \in B_{p, q}^{s}(\mathbb{R}) \Longleftrightarrow \sum_{j \geq 3}\left(2^{j s}\left\|\phi \sum_{k=1}^{k(j)} \Theta_{j, k}\right\|_{L_{p}(\mathbb{R})}\right)^{q}<\infty .
$$

(Standard modification if $q=\infty$.)

On the other hand we have (the last $\approx_{j}$ is valid only if $k(j) \neq 0$, this means $j$ sufficiently large)

$$
\left\|\phi \sum_{k=1}^{k(j)} \Theta_{j, k}\right\|_{L_{p}(\mathbb{R})} \approx_{j}\left(\frac{1}{(\ln j)^{p}} k(j) 2^{-j}\right)^{\frac{1}{p}} \approx_{j} \frac{1}{\ln j} 2^{-j \frac{\lambda}{p}} .
$$

(ii) For $n \in \mathbb{N} \backslash\{1\}, 0<q \leq \infty$ and $s \in \mathbb{R}$ and we have the equivalence $\Theta \in B_{p, q}^{s}\left(\mathbb{R}^{n}\right) \Longleftrightarrow \sum_{j \geq 3}\left(2^{j s}\left\|\phi \sum_{k=1}^{k(j)} \sum_{l=1}^{d(j)^{n-1}} \Theta_{j, k, l}\right\|_{L_{p}\left(\mathbb{R}^{n}\right)}\right)^{q}<\infty$. (Standard modification if $q=\infty$.)

On the other hand we have (if $j$ sufficiently large)

$$
\left\|\phi \sum_{k=1}^{k(j)} \sum_{l=1}^{d(j)^{n-1}} \Theta_{j, k, l}\right\|_{L_{p}\left(\mathbb{R}^{n}\right)} \approx_{j}\left(\frac{1}{(\ln j)^{p}} k(j) d(j)^{n-1} 2^{-j n}\right)^{\frac{1}{p}} \approx_{j} \frac{1}{\ln j} 2^{-j \frac{h+\lambda}{p}} .
$$

Proof of Theorem 5.9. We will restrict us to the case $n=1$. For $n \in \mathbb{N} \backslash\{1\}$ the proof is analogous, although with calculations slightly more complicated. In corresponding parts (i) and (ii) we use essentially the fact that the case $n=1$ remains valid if we replace $\Lambda$ by $\Lambda\left(., x_{2}, \ldots, x_{n}\right)$, for fixed $\left(x_{2}, \ldots, x_{n}\right) \in \mathbb{R}^{n-1}$. For the convenience of the presentation we assume $0<\lambda<1$, however the case $\lambda=1$ can be dealt similarly. Moreover, 
the proof of parts (a) and (c) are similar to the corresponding ones in the proof of Theorem 5.8.

We observe that $2^{-(j+1) \lambda} \leq 2^{-j \lambda}-k 2^{-j} \leq 2^{-j \lambda}-2^{-j}, \forall k=1, \ldots, k(j)$, for all $j \in \mathbb{N}$. We make use of the estimation $|x|^{m}|\psi(x)| \leq c_{m}$ for all $m \in \mathbb{N}_{0}$.

(i) We have uniform convergence of the series $\Lambda=\sum_{j \geq 1} \sum_{k=1}^{k(j)} \Lambda_{j, k}$ in all $\mathbb{R}$.

Then $\Lambda$ is bounded and continuous.

(i1) In fact, with the same techniques used in part (i1) of the proof of Theorem 5.6, we can show the uniform convergence of $\sum_{j \geq 1} \sum_{k=1}^{k(j)}\left|\Lambda_{j, k}\right|$ on $(-\infty, 0]$.

(i2) Also, with the same techniques used in parts (i2) and (i3) of the proof of Theorem 5.6, we can show the uniform convergence of $\sum_{j \geq 1} \sum_{k=1}^{k(j)}\left|\Lambda_{j, k}\right|$ on $(0, \infty)$.

(ii) Let us prove the part (b) for $n=1$ and $0<\lambda<1$. Let $j \in \mathbb{N}, \rho \geq 0$ and $C^{\rho}=\{t \in \mathbb{R}:|t| \geq \rho\}$. We define $\sum_{j}^{\rho}$ as a general sum over all intervals $I \cap C^{\rho}$ with $I$ as in Definition 3.1 and $|I|=2^{-j}$. Then, for $r \in \mathbb{N}_{0}$ and $\rho \geq 0$, there is a positive constant $c_{r}$ independent of $j$ and $\rho$ such that

$$
\rho^{r} \sum_{j}^{\rho} \operatorname{osc}(\psi) \leq c_{r} .
$$

Here we consider $0^{0}=1$. In order to prove (1), we notice that the sum of the left hand side can be estimated from above by $\rho^{r} \sum_{j}^{\rho} \sup \left|\psi^{\prime}\right| 2^{-j} \leq 2^{-j} \sum_{|I|=2^{-j}} \sup _{I}\left|t^{r} \psi^{\prime}(t)\right| \leq 2^{-j} \sum_{\tau \geq 1} g_{r}\left(\tau 2^{-j}\right)$, where $g_{r}(t)=\frac{c_{r} t^{r}}{1+t^{r+2}}, \forall t \geq 0$. Let $m_{r} \in \mathbb{N}$ such that $g_{r}$ is decreasing on $\left[m_{r}, \infty\right)$. Then $\rho^{r} \sum_{j}^{\rho} \operatorname{osc}(\psi) \leq 2^{-j} \sum_{\tau=1}^{2^{j} m_{r}} \sup g_{r}+2^{-j} \sum_{\tau>2^{j} m_{r}} g_{r}\left(\tau 2^{-j}\right) \leq$ $c_{r}+\int_{m_{r}}^{\infty} g_{r}(t) d t=c_{r}^{\prime}$, which proves (1).

We define $C^{\rho, u, v}=\{t \in \mathbb{R}:|t-m(u, v)| \geq \rho\}$ and $S_{j, u, v}^{\rho, u, v}=$ $\sum_{j}^{\rho, u, v} \operatorname{osc}\left(\Lambda_{u, v}\right), \forall u \in \mathbb{N}, \forall v \in\{1, \ldots, k(u)\}$, where the sum $\sum_{j}^{\rho, u, v}$ is taken over all intervals $I \cap C^{\rho, u, v}$ with $I$ as in Definition 3.1 and $|I|=2^{-j}$. Then by formula (1) there is a positive constant $c_{r}$ 
independent of $j, \rho, u$ and $v$ such that

$$
\left(2^{u} \rho\right)^{r} S_{j, u, v}^{\rho, u, v} \leq c_{r}
$$

Let $S_{j, u, v}=\sum_{|I|=2^{-j}} \operatorname{osc}_{I}\left(\Lambda_{u, v}\right), \forall u \in \mathbb{N}, \forall v \in\{1, \ldots, k(u)\}$. Of course we have

$$
\sum_{|I|=2^{-j}}{ } \operatorname{sc}_{I}(\Lambda) \leq \sum_{u \geq 1} \sum_{v=1}^{k(u)} S_{j, u, v} .
$$

Let $\sum_{j}^{\prime}, \sum_{j}^{\prime \prime}$ and $\sum_{j}^{\prime \prime \prime}$ be general sums over all intervals $I \cap C$ with $I$ as in Definition 3.1 and $|I|=2^{-j}$, where $C$ represents the set $(-\infty, 0]$, $\left[0,2^{-j \lambda}\right]$ and $\left[2^{-j \lambda}, \infty\right)$, respectively.

(ii1) By formula (2) and calculations like in (i1) for $x \leq 0$, but with $S_{j, u, v}^{|x-m(u, v)|, u, v}$ in place of $\left|\Lambda_{u, v}(x)\right|$, we have the inequality $\sum_{u \geq 1} \sum_{v=1}^{k(u)} S_{j, u, v}^{\prime} \leq c$, where by definition $S_{j, u, v}^{\prime}$ is obtained from $S_{j, u, v}$ by replacing $\sum_{|I|=2^{-j}}$ by $\sum_{j}^{\prime}$. Therefore, by the obvious counterpart of formula (3) for $\sum_{j}^{1}$, we have the estimation $\sum_{j}^{\prime} \operatorname{osc}(\Lambda) \leq c$.

(ii2) Because $\Lambda$ is bounded, we have the estimation $\sum_{j}^{\prime \prime} \operatorname{osc}(\Lambda) \leq$ $c 2^{j(1-\lambda)}$.

(ii3) Also as counterpart of formula (3) we have the inequality $\sum_{j}^{\prime \prime \prime} \operatorname{osc}(\Lambda) \leq \sum_{u \geq 1} \sum_{v=1}^{k(u)} S_{j, u, v}^{\prime \prime \prime}$, where by definition $S_{j, u, v}^{\prime \prime \prime}$ is obtained from $S_{j, u, v}$ by replacing $\sum_{|I|=2^{-j}}$ by $\sum_{j}^{\prime \prime \prime}$.

We denote by $\sum_{j}^{\prime \prime \prime *}$ or $\sum_{j}^{\prime \prime \prime * *}$ the right hand side of the this inequality, if we make the restriction $1 \leq u \leq j$ or $u>j$, respectively. Then, by formula (2) and calculations like in (i2) for $x \geq 2^{-j \lambda}$, but with $S_{j, u, v}^{|x-m(u, v)|, u, v}$ in place of $\left|\Lambda_{u, v}(x)\right|$, we have the estimation $\sum_{j}^{\prime \prime \prime * *} \leq c$. 
On the other hand, as a particular case of formula (1) with 0 in place of $\rho$ and $r$, we have $\sum_{|I|=2^{-j}} \operatorname{osc}_{I}(\psi) \leq c$, where $c$ is independent of $j$. Then we have the inequality $\sum_{j}^{\prime \prime \prime *} \leq \sum_{u=1}^{j} k(u) c \leq$ $c^{\prime} 2^{j(1-\lambda)}$, and therefore the estimation $\sum_{j}^{\prime \prime \prime} \operatorname{osc}(\Lambda) \leq c 2^{j(1-\lambda)}$.

(ii4) Hence by (ii1), (ii2) and (ii3) we obtain $\operatorname{Osc}(\nu, \Lambda) \leq c 2^{j(1-\lambda)}$. Then $\Lambda \in V^{\lambda}(\mathbb{R})$ and by the proof of Theorem 4.2 we have also $\Lambda_{0} \in V^{\lambda}(\mathbb{R})$, so Theorem 4.1 gives $\overline{\operatorname{dim}}_{B} \Gamma\left(\Lambda_{0}\right) \leq 2-\lambda$.

(ii5) Finally, with the same argument given for the proof of part (c) we have also $\Lambda_{0} \in \underline{B}_{p}^{\frac{\lambda}{p} *}\left(\mathbb{R}^{n}\right)$ for $0<p \leq \infty$. Hence Remark 5.5-(a) with 1 in place of $p$ gives $\underline{\operatorname{dim}}_{B} \Gamma\left(\Lambda_{0}\right) \geq 2-\lambda$. Therefore we have $\operatorname{dim}_{B} \Gamma\left(\Lambda_{0}\right)=2-\lambda$.

\section{References}

[1] A. Carvalho, Box dimension and smoothness in function spaces, (2004), preprint.

[2] A. Deliu and B. Jawerth, Geometrical dimension versus smoothness, Constr. Approx., 8 (1992), 211-222.

[3] K. Falconer, Fractal Geometry, John Wiley, Chichester, 1990.

[4] S. Jaffard, Oscillation spaces: Properties and applications to fractal and multifractal functions, J. Math. Phys., 39 (1998), 4129-4141.

[5] S. Jaffard, Wavelet expansions, function spaces and multifractal analysis, Math. Phys. Chem., 33 (2001), 127-144.

[6] A. Kamont and B. Wolnik, Wavelet expansions and fractal dimension, Constr. Approx., 15 (1999), 97-108.

[7] V. Khoan, Distributions, Analyse de Fourier, Opérateurs aux Dérivées Partielles, tome 2, Librairie Vuibert, Paris, 1972.

[8] M. Solomyak, Piecewise-polynomial approximation of functions from Sobolev spaces, revisited, M. University. Discourses Math. Appl., 3 (1996) $7-27$.

[9] H. Triebel, Theory of Function Spaces, Birkhäuser, Basel, 1983.

[10] H. Triebel, Fractals and Spectra, Birkhäuser, Basel, 1977.

[11] H. Triebel, The Structure of Functions, Birkhäuser, Basel, 2001.

[12] H. Triebel, Fractal characteristics of measures; an approach via function spaces, Journ. Fourier Anal. Appl., 9 (2003) 411-430. 
[13] H. Triebel, A note on wavelet bases in function spaces, Orlicz Centenary Vol. Banach Center Publ., 64 (2004) 193-205.

Departamento de Matemática of Universidade de Aveiro 3810-193 Aveiro

Portugal

(E-mail : abel@mat.ua.pt) 


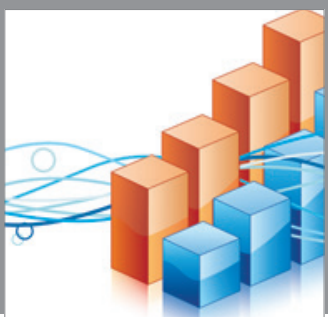

Advances in

Operations Research

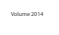

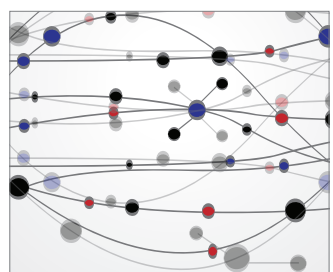

\section{The Scientific} World Journal
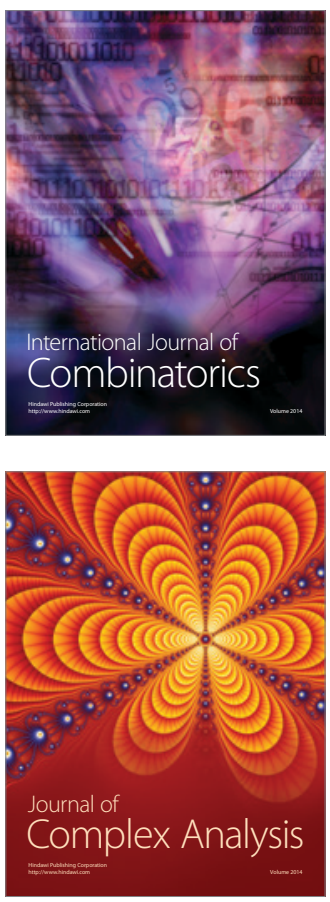

International Journal of

Mathematics and

Mathematical

Sciences
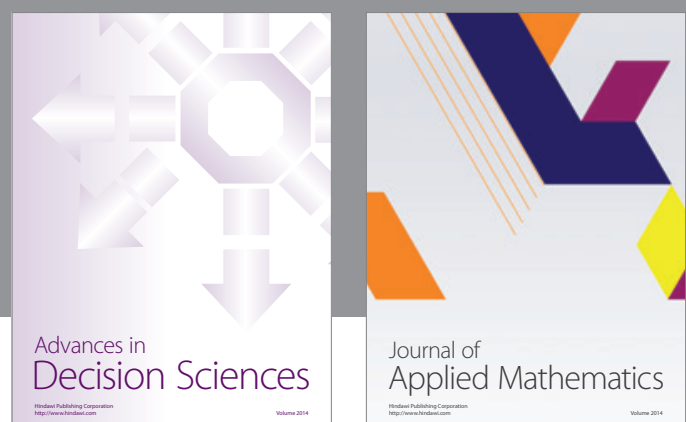

Journal of

Applied Mathematics
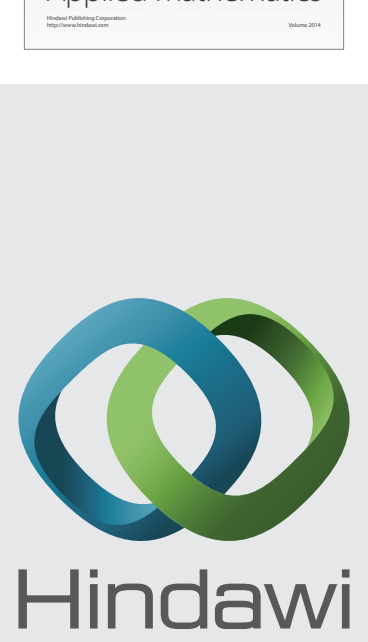

Submit your manuscripts at http://www.hindawi.com
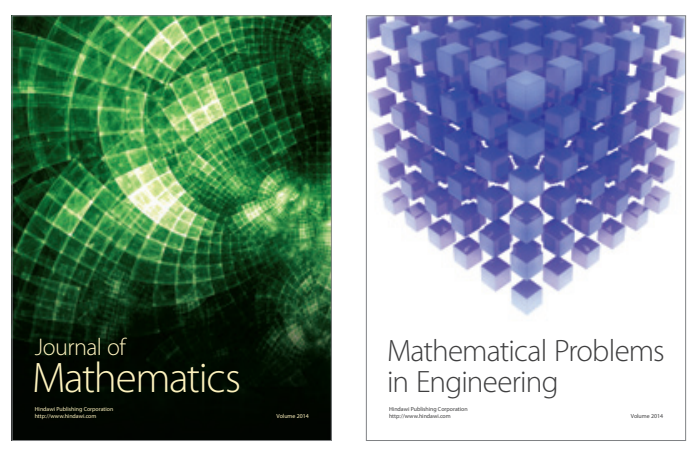

Mathematical Problems in Engineering
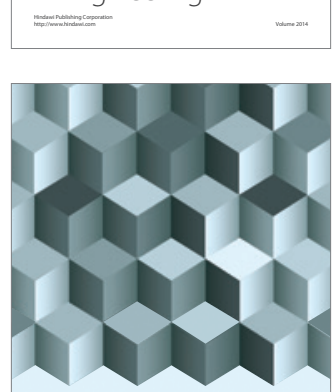

Journal of

Function Spaces
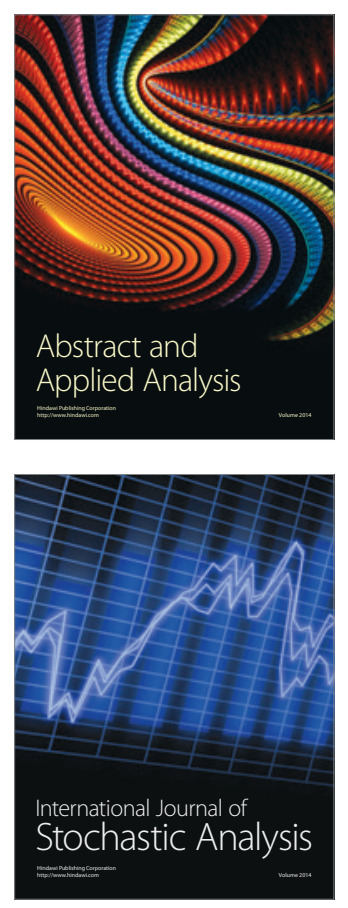

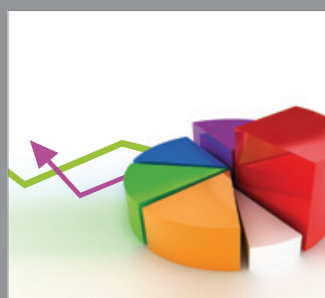

ournal of

Probability and Statistics

Promensencen
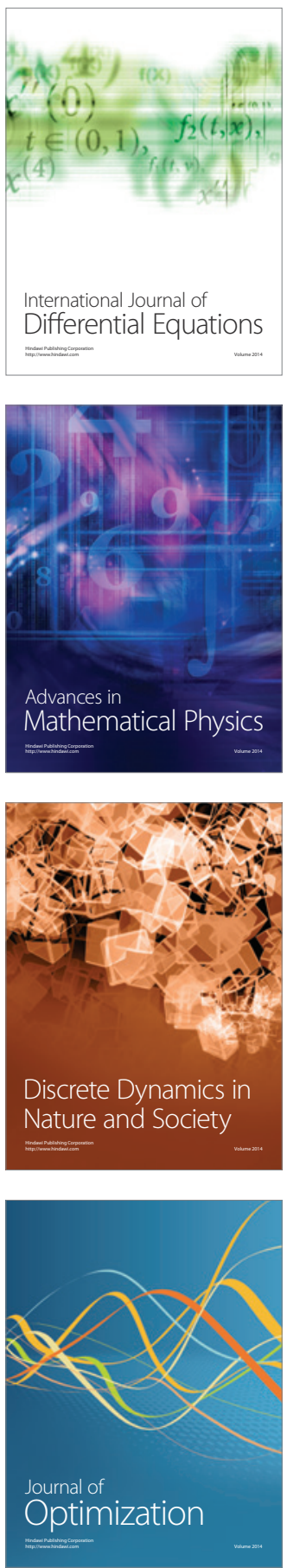